\title{
Predictions of rainfall-runoff response and soil moisture dynamics in a microscale catchment using the CREW model
}

\author{
H. Lee ${ }^{1}$, E. Zehe ${ }^{2}$, and M. Sivapalan ${ }^{3}$ \\ ${ }^{1}$ School of Environmental Systems Enineering, The University of Western Australia, 35 Stirling Highway, Crawley, WA \\ 6009, Australia \\ ${ }^{2}$ Institute of Geoecology, University of Potsdam, Germany \\ ${ }^{3}$ Departments of Geography \& Civil and Environmental Engineering, University of Illinois at Urbana-Champaign, \\ 220 Davenport Hall, 607 S. Mathews Avenue, Urbana, IL 61801, USA
}

Received: 23 January 2006 - Published in Hydrol. Earth Syst. Sci. Discuss.: 17 July 2006

Revised: 5 December 2006 - Accepted: 24 January 2007 - Published: 5 February 2007

\begin{abstract}
Predictions of catchment hydrology have been performed generally using either physically based, distributed models or conceptual lumped or semi-distributed models. In recognition of the disadvantages of using either of these modeling approaches, namely, detailed data requirements in the case of distributed modeling, and lack of physical basis of conceptual/lumped model parameters, Reggiani et al. (1998, 1999) derived, from first principles and in a general manner, the balance equations for mass, momentum and energy at what they called the Representative Elementary Watershed (or REW) scale. However, the mass balance equations of the REW approach include mass exchange flux terms which must be defined externally before their application to real catchments. Developing physically reasonable "closure relations" for these mass exchange flux terms is a crucial pre-requisite for the success of the REW approach. As a guidance to the development of closure relations expressing mass exchange fluxes as functions of relevant state variables in a physically reasonable way, and in the process effectively parameterizing the effects of sub-grid or sub-REW heterogeneity of catchment physiographic properties on these mass exchange fluxes, this paper considers four different approaches, namely the field experimental approach, a theoretical/analytical approach, a numerical approach, and a hybrid approach combining one or more of the above. Based on the concept of the scaleway (Vogel and Roth, 2003) and the disaggregation-aggregation approach (Viney and Sivapalan, 2004), and using the data set from Weiherbach catchment in Germany, closure relations for infiltration, exfiltration and groundwater recharge were derived analytically, or on theoretical grounds, while numerical experiments with a detailed fine-scale, distributed
\end{abstract}

Correspondence to: $\mathrm{H}$. Lee

(haksu.lee@noaa.gov) model, CATFLOW, were used to obtain the closure relationship for seepage outflow. The detailed model, CATFLOW, was also used to derive REW scale pressure-saturation (i.e., water retention curve) and hydraulic conductivity-saturation relationships for the unsaturated zone. Closure relations for concentrated overland flow and saturated overland flow were derived using both theoretical arguments and simpler process models. In addition to these, to complete the specification of the REW scale balance equations, a relationship for the saturated area fraction as a function of saturated zone depth was derived for an assumed topography on the basis of TOPMODEL assumptions. These relationships were used to complete the specification of all of the REW-scale governing equations (mass and momentum balance equations, closure and geometric relations) for the Weiherbach catchment, which are then employed for constructing a numerical watershed model, named the Cooperative Community Catchment model based on the Representative Elementary Watershed approach (CREW). CREW is then used to carry out sensitivity analyses with respect to various combinations of climate, soil, vegetation and topographies, in order to test the reasonableness of the derived closure relations in the context of the complete catchment response, including interacting processes. These sensitivity analyses demonstrated that the adopted closure relations do indeed produce mostly reasonable results, and can therefore be a good basis for more careful and rigorous search for appropriate closure relations in the future. Three tests are designed to assess CREW as a large scale model for Weiherbach catchment. The first test compares CREW with distributed model CATFLOW by looking at predicted soil moisture dynamics for artificially designed initial and boundary conditions. The second test is designed to see the applicabilities of the parameter values extracted from the upscaling procedures in terms of their

Published by Copernicus GmbH on behalf of the European Geosciences Union. 
ability to reproduce observed hydrographs within the CREW modeling framework. The final test compares simulated soil moisture time series predicted by CREW with observed ones as a way of validating the predictions of CREW. The results of these three tests, together, demonstrate that CREW could indeed be an alternative modelling framework, producing results that are consistent with those of the distributed model CATFLOW, and capable of ultimately representing processes actually occurring at the larger scale in a physically sound manner.

\section{Introduction}

Ability to make hydrological predictions has become an essential part of sustainable management of water resources, water quality and water related natural hazards, especially in environments where climatic or human induced land use changes are under way. Catchments undergoing a transition from one state to a different state through climatic or land use changes can be considered as ungauged basins, due to the fact that under conditions of change, past measurements or gauging are poor or inadequate indicators of the future. The global, decadal initiative on predictions in ungagued basins or PUB (Sivapalan et al., 2003) has been designed to address this as yet unsolved problem in hydrology. To address the problem of PUB, and in particular, to predict the effects of climatic and land use changes, it is increasingly necessary to develop hydrological models that are based on a deeper level of process understanding rather than merely rely on calibrations carried out with past observations. For hydrological predictions in meso-scale catchments, the usual practice is to use so-called conceptual models, which can be lumped or quasi-distributed due to their efficiency in terms of data requirements and computational costs, traits that put them at a considerable advantage compared to physically based, fully distributed models, notwithstanding the sound theoretical basis of the latter-type models. Parameters used in lumped or quasi-distributed conceptual models often have very little physical meaning in the traditional sense, due to the lack of a physically-based theory at the catchment scale, and consequently these parameters cannot be estimated unambiguously in the field or from field data. Therefore, conceptual models will be inadequate to address PUB problems in an efficient or physically sound manner. To deal with the PUB problem, the chosen model must be flexible enough to incorporate new findings about processes in changed environments and new ways of capturing them in models. In addition, parameters of the model must be capable of being estimated from field data and of reflecting likely environmental changes, and their meanings must be sound enough on physical grounds. In order to make better predictions and reduce predictive uncertainties, the chosen model must have a holistic model structure that incorporates changes in the environ- ment in a consistent manner so as to reduce model structure uncertainties.

Recently, Reggiani et al. $(1998,1999)$ proposed a new hydrological modeling framework based on balance equations for mass, force and energy, derived directly at the scale of what they called the Representative Elementary Watershed (REW). The REW approach presents, potentially, a novel framework for developing hydrological models directly at the catchment scale, in a physically based and also physically meaningful manner. The REW is a hydrologically significant control volume that lends itself to thermodynamically consistent volume averaging. Due to the R (representative) aspect of the REW this implies essentially that the REW is a means of separating scales. It must therefore be larger than the characteristic length scales of all relevant hydrologic landscape properties. At the mesoscale the REW approach offers, in principle, several advantages over traditional (lumped or quasi-distributed) conceptual models, and over the current generation of physically based, fully distributed (grid based) models. Firstly, the equations derived as part of the REW approach address (volume) average dynamics directly at REW scale, as opposed to the current generation of distributed models that operate at the representative elementary volume (REV) scale. Therefore, models based on the the REW scale balance equations remain modest in terms of both their computational burden and their input and parameter requirements. Secondly, the REW scale balance equations have been derived in a comprehensive manner for the whole catchment or REW, as opposed to being derived separately for different processes, as is the case with many traditional distributed models. Special care has been taken to respect not only the individual component processes, but also the various process interactions amongst parts of the REW. This enhances the holistic nature of the REW approach for characterizing overall catchment responses. Thirdly, by being general and not tied to specific process formulations, e.g., about how to describe mass and/or momentum exchanges at the REW scale, the REW approach can easily benefit from further advances in process understanding and process descriptions emerging from new field experiments carried out at the hillslope or REW/catchment scale. However, this is also the most difficult hurdle to be taken when building new models based on the REW approach, because process descriptions at that scale are in general not widely available (Beven, 2002).

The "heart" of the REW approach is the set of coupled mass and force balance equations for "different zones" within an REW, such as the unsaturated zone, the saturated zone and the channel zone. However, mass fluxes between these different zones are generally unknown, with the result that there are more unknowns than there are balance equations, making the set of balance equations indeterminate. In analogy to turbulence theory this is called the "closure problem". Closure means essentially the development of physically reasonable process formulation for the vari- 
ous mass exchange fluxes that incorporate the effects of subREW scale spatial heterogeneities, and expressed in terms of selected REW scale state variables and catchment characteristics. A related problem is the derivation of REW scale constitutive relations that relate one or more state variables amongst themselves, e.g. REW scale capillary pressure vs saturation and hydraulic conductivity vs saturation relationships in the unsaturated zone, again incorporating the effects of sub-REW scale heterogeneities. The latter is essential to describe capillary rise and recharge at that scale.

To a certain extent, the closure relations can be a result of an upscaling of process descriptions available at the point or REV scale, towards physically reasonable process parameterizations appropriate to the REW scale. In fact, they could be much more than this, and could represent processes that occur at the larger (e.g., REW) scale, and requiring descriptions that transcend familiar small scale ones, in which case upscaling may not be adequate. In either case, theory alone, of the sort used in the derivation of the REW scale balance equations and constitutive theory, i.e., Newton's laws of motion and the 2nd law of thermodynamics, is not sufficient to generate these. Closure relations and constitutive relations represent the hydrological functioning of catchment/landscape compartments at the REW scale. Hence, they are always related to a catchment/landscape, process and equations and have to be determined empirically, by "looking" at the catchment/landscape of interest. Ideally, they will have to be estimated from experiments in the field that operated directly at the REW scale. However, as such data are out of reach yet, one should at least employ field data that are currenty state of the art and combine these with appropriate integration of assumed, measured or simulated realistic patterns of sub-grid, or sub-REW, heterogeneity. Indeed, the closure relations are the best mechanism to ground the REW theory to reality, through physically realistic and reasonable descriptions of actual hydrological processes and their underlying physical mechanisms, expressed in terms of parameterizations involving landscape and climatic properties. Therefore, they are also intimately connected to the issue of estimation model parameters (although this link and the related equifinality problem are not explored in this paper). To summarize, the applicability of the REW approach to model real world catchments stands and falls with the assessment of closure relations and assessment the related parameters/constitutive relations that are valid at the REW scale (Beven, 2002; Reggiani and Schellekens, 2003).

The chief focus of this paper is on the derivation and assessment of various closure relations and constitutive relations for Weiherbach, a micro-scale catchment located in south-west Germany, which has been well studied for more than 10 years. This catchment offers an extensive hydrological data set, including soil hydraulic functions and soil patterns, precipitation data, meteorological data, and distributed soil moisture observations and tracer data (Zehe et al., 2001). In addition, a detailed process model, CATFLOW, is also available, having been previously developed at this catchment and shown to closely portray all aspects of systems dynamics at hillslope and catchment scales. Within a 1.5 year simulation period the model predicted observed discharges with a Nash-Sutcliffe efficiency of 0.82 and at the same time predicted point observations of evapotranspiration with a correlation of 0.92 and point observations of soil moisture at 61 locations with correlation coefficients of between 0.65 to 0.75 (Zehe et al., 2001; Zehe and Blöschl, 2004). Hence this model may be employed for deriving closure relations through conducting of carefully targeted numerical experiments. The objective is to explore this and alternative approaches currently available and report on the progress made so far in developing closure relations for this place. We will then present the resulting complete set of coupled balance equations for mass and momentum, and the associated geometric relations, followed by a sensitivity analysis with the resulting complete REW-scale hydrological model, which we call CREW. With the complete REW scale model (CREW) we will simulate the water balance of the Weiherbach catchment using observed rainfall and potential evaporation time series and compare model predictions of hydrological response to both corresponding observations and also the integrated predictions of CATFLOW obtained for the same period. The latter comparison will illustrate, in particular, whether the simulations of the internal state dynamics predicted by the CREW are comparable with the integral dynamics simulated with the fully distributed model (CATFLOW), which can be deemed to be the best guess of how the true integrated soil moisture in the Weiherbach catchment may have evolved during the simulation period.

\section{The REW-approach and the Study Area}

\subsection{The REW approach as foundation for meso-scale mod- els}

An REW is taken as the smallest resolvable spatial unit of a meso-scale watershed, and is composed of five zones: unsaturated zone (u-zone), saturated zone (s-zone), concentrated overland flow zone (c-zone), saturated overland flow zone (ozone), and channel zone (r-zone). These are delineated based on known physical characteristics of typical watersheds, and on characteristic time scales that are typical of various hydrological processes (Reggiani et al., 1998) through averaging. Hence, an REW can be deemed to separate the next hydrological significant scale. The mass, energy and momentum balances within the individual zones of the REW are described using a coupled set of ordinary differential equations, derived from thermodynamic principles, by means of averaging. Figure 1a presents the schematic of a typical watershed that is discretized into three REWs based on the geometry of channel network, and Fig. 1b illustrates the sub-regions making up the REW, and the mass exchange fluxes between 
different sub-regions of each REW, and those between different REWs. A simpler set of REW-scale balance equations of mass and momentum applicable to these REWs and their sub-regions, from those first derived by Reggiani et al. (1998, 1999) is used in the rest of this paper. These are presented in Eqs. (1) to (11) below. For further details regarding their derivation and the meaning of the variables, the reader is referred to Reggiani et al. $(1998,1999,2000)$, and the nomenclature given at the end of the paper.

$$
\underbrace{\frac{d}{d t}\left(\varepsilon y^{s} \omega^{s}\right)}_{\text {storage }}=\underbrace{e^{s o}}_{\text {seepage }}+\underbrace{e^{s u}}_{\text {exchag. with unsat. zone }}+\underbrace{e^{s r}}_{\text {sat. zone-river exchange }}+\underbrace{\sum_{l} e_{l}^{s A}+e_{\text {ext }}^{s A}}_{\text {exchange across mantle segments }}
$$

$\underbrace{\frac{d}{d t}\left(\varepsilon y^{u} \omega^{u} s^{u}\right)}_{\text {storage }}=\underbrace{e^{u c}}_{\text {infiltration }}+\underbrace{e^{u s}}_{\text {exchag. with sat. zone }}+\underbrace{e_{w g}^{u}}_{\text {evaporation }}+\underbrace{\sum_{l} e_{l}^{u A}+e_{\text {ext }}^{u A}}_{\text {exchange across mantle segments }}$

$\underbrace{\frac{d}{d t}\left(y^{c} \omega^{c}\right)}_{\text {storage }}=\underbrace{e^{c u}}_{\text {infiltration into unsat. zone }}+\underbrace{e^{c o}}_{\text {flow to sat. overl. flow }}+\underbrace{e^{c t o p}}_{\text {rainfall or evaporation }}$

$\underbrace{\frac{d}{d t}\left(y^{o} \omega^{o}\right)}_{\text {storage }}=\underbrace{e^{o r}}_{\text {lat. channel inflow }}+\underbrace{e^{o s}}_{\text {seepage }}+\underbrace{e^{o c}}_{\text {inflow from conc. overl. flow }}+\underbrace{e^{o t o p}}_{\text {rainfall or evaporation }}$

$\underbrace{\frac{d}{d t}\left(m^{r} \xi^{r}\right)}_{\text {storage }}=\underbrace{e^{r o}}_{\text {lateral inflow }}+\underbrace{e^{r s}}_{\text {channel-sat. zone exch. }}+\underbrace{\sum_{l} e_{l}^{r A}+e_{\text {ext }}^{r A}}_{\text {inflow, outflow }}+\underbrace{e^{r t o p}}_{\text {rainfall or evaporation }}$

$$
\begin{aligned}
& \underbrace{ \pm \sum_{l} A_{l, \lambda}^{s A}\left[-p^{s}+\rho\left(\phi_{l}^{s A}-\phi^{s}\right)\right]}_{\text {inter-REW driving force }}+\underbrace{ \pm A_{\text {ext, }}^{s A}\left[-p^{s}+\rho\left(\phi_{\text {ext }}^{s A}-\phi^{s}\right)\right]}_{\text {force acting on the external boundary }}+\underbrace{ \pm A_{\lambda}^{s b o t}\left[-p^{s}+\rho\left(\phi^{s b o t}-\phi^{s}\right)\right]}_{\text {force at the bottom boundary }} \\
& \quad=\underbrace{-R^{s} v_{\lambda}^{s}}_{\text {resistance to flow }} ; \lambda=x, y
\end{aligned}
$$

$\underbrace{ \pm \sum_{l} A_{l, \lambda}^{u A}\left[-p^{u}+\rho\left(\phi_{l}^{u A}-\phi^{u}\right)\right]}_{\text {inter-REW driving force }}+\underbrace{ \pm A_{\text {ext, }}^{u A}\left[-p^{u}+\rho\left(\phi_{\text {ext }}^{u A}-\phi^{u}\right)\right]}_{\text {force acting on the external boundary }}=\underbrace{-R^{u} v_{\lambda}^{u}}_{\text {resistance to flow }} ; \lambda=x, y$

$\underbrace{\left[-p^{u}+\rho\left(\phi^{u c}-\phi^{u}\right)\right] \varepsilon \omega^{u}}_{\text {force top }}-\underbrace{\rho \varepsilon s^{u} y^{u} \omega^{u} g}_{\text {gravity }}=\underbrace{-R^{u} v_{z}^{u}}_{\text {resistance force }}$

$\underbrace{\left(\rho y^{c} \omega^{c}\right) \frac{d v^{c}}{d t}}_{\text {inertialterm }}-\underbrace{\rho y^{c} \omega^{c} g \sin \gamma^{c}}_{\text {gravity }}=\underbrace{-U^{c} v^{c}\left|v^{c}\right|}_{\text {resistance to flow }}$ 


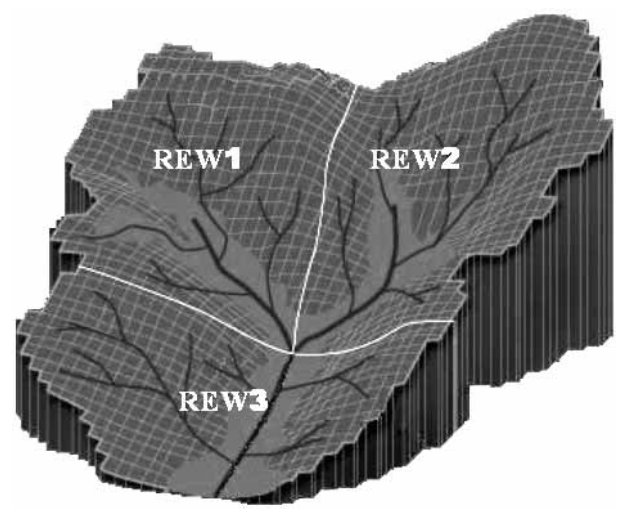

(a)

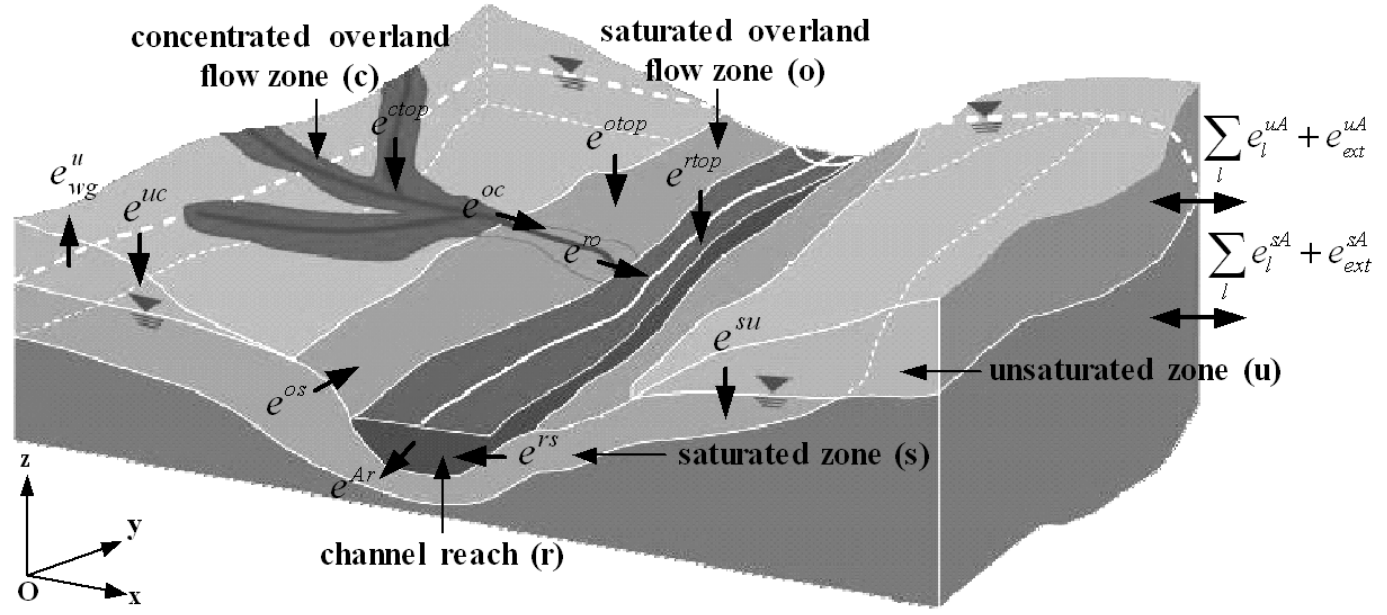

(b)

Fig. 1. (a) Catchment discretization into 3 REW units (b) Exchanging mass fluxes and subregions making up the spatial domain of a REW after Reggiani et al. $(1999,2000)$ : $e^{u c}$ denotes infiltration, $e_{w g}^{u}$ evapotranspiration from unsaturated zone, $e^{s u}$ recharge or capillary rise, $e^{\text {ctop }}, e^{\text {otop }}$ and $e^{\text {rtop }}$ rainfall or evaporation at $\mathrm{c}$, o and r-zones respectively, $e^{o c}$ concentrated overland flow, $e^{\text {ro }}$ saturated overland flow, $e^{o s}$ seepage flow, $e^{r s}$ flow from saturated zone to channel, $e^{A r}$ channel flow at outlet, and $\sum_{l} e_{l}^{u A}+e_{\text {ext }}^{u A}$ and $\sum_{l} e_{l}^{s A}+e_{\text {ext }}^{s A}$ mass exchange across mantle segment at $\mathrm{u}$ and s-zones respectively.

$\underbrace{\left(\rho y^{o} \omega^{o}\right) \frac{d v^{o}}{d t}}_{\text {inertialterm }}-\underbrace{\rho y^{o} \omega^{o} g \sin \gamma^{o}}_{\text {gravity }}=\underbrace{-U^{o} v^{o}\left|v^{o}\right|}_{\text {resistance to flow }}$

$\underbrace{\left(\rho m^{r} \xi^{r}\right) \frac{d v^{r}}{d t}}_{\text {inertial term }}=\underbrace{\rho g m^{r} \xi^{r} \sin \gamma^{r}}_{\text {gravitational force }}-\underbrace{U^{r} v^{r}\left|v^{r}\right|}_{\text {Chezy resistance }}+\underbrace{ \pm \sum_{l} A_{l}^{r A} \cos \delta_{l}\left[-p^{r}+\rho\left(\phi_{l}^{r A}-\phi^{r}\right)\right]}_{\text {pressure forces exchanged among REWs }}+\underbrace{A_{\text {ext }}^{r A}\left[-p^{r}+\rho\left(\phi_{\text {ext }}^{r A}-\phi^{r}\right)\right]}_{\text {pressure force at watershed outlet }}$ 
where $\varepsilon$ is porosity, $y^{i}(i=u, s, c, o, r)$ is the average vertical thickness of the $i$ subregion, $\omega^{i}(i=u, s, c, o, r)$ is the time averaged surface area fraction of the $i$ subregion, $s^{u}$ is the saturation degree of the unsaturated zone, $m^{r}$ is the channel cross sectional area, $\xi^{r}$ is the drainage density, $e^{i j}$ ( $i$ or $j=u, s, c, o, r)$ the rate of water mass exchange between the $i$ and $j$ subregions, $e_{w g}^{u}$ is the rate of evapotranspiration from the unsaturated zone, $e_{l}^{j A}(j=u, s, r)$ is the rate of water mass exchange from the $j$ subregion across the $l$ th mantle segment, $e_{\text {ext }}^{j A}(j=u, s, r)$ is the water mass exchange from the $j$ subregion across the external watershed boundary, $A_{l}^{j A}$ and $A_{\text {ext }}^{j A}(j=u, s, r)$ are the mantle surface with horizontal normal delimiting the REW externally at the $j$ subregion with the $l$ th mantle segment and the external watershed boundary, respectively, $A^{s b o t}$ is the mantle surface corresponding to the bottom part of the saturated zone, $p^{i}(i=u, s, r)$ is the pressure of the $i$ subregion, $\rho$ is water mass density, $\phi^{i}, \phi^{s b o t}$, $\phi_{l}^{i A}$, and $\phi_{\text {ext }}^{i A}(i=u, s, r)$ are the gravitational potential at the $i$ subregion, at the bottom part of the saturated zone, at the interface of the $i$ subregion and $l$ th mantle segment, and at the interface of the $i$ subregion and the external watershed boundary, respectively, $v^{i}(i=u, s, c, o, r)$ is the water velocity within $i$ subregion, $v_{z}^{u}$ is the vertical water velocity within the unsaturated zone, $g$ is gravitational acceleration, $R^{i}(i=u$, $s)$ is the first order friction term of the $i$ subregion, $U^{i}(i=c$, $o, r)$ is the second order friction term of the $i$ subregion, $\gamma^{i}$ $(i=c, o, r)$ is the slope angle of the $i$ subregion flow plane with respect to the horizontal plane, and $\delta_{l}$ is the local angle between the reach of the $l$ th REW and the reach of the REW of interest.

To summarize, Eqs. (1) to (5) represent, respectively, mass balance of the saturated zone (s-zone), the unsaturated zone (u-zone), the concentrated overland flow zone (c-zone), the saturated overland flow zone (o-zone), and the channel reach (r-zone). Equations (6) to (11) represent momentum balance of the saturated zone, unsaturated zone in the horizontal direction, unsaturated zone in the vertical direction, concentrated overland flow zone, saturated overland flow zone and the channel reach. In Eqs. (6) and (7), the signs are either positive or negative according to the orientation of $A_{l}^{j A}, A_{\text {ext }}^{j A}$ $(j=u, s)$ and $A^{s b o t}$ with respect to the reference system, while the sign of the second-last term in Eq. (11) is positive for the outlet sections and negative for the inlet sections of the channel reaches (Reggiani et al., 1999).

\subsection{The closure problem}

The $e^{i j}$ terms in the mass balance Eqs. (1) to (5), also shown in Fig. 1b, represent mass exchange fluxes between the $i$ and $j$ sub-regions such as infiltration, bare soil evaporation and transpiration by root uptake, groundwater recharge/capillary rise, saturated and concentrated overland flow, seepage outflow, and channel flow. These fluxes are generally unknown, and must be externally specified. Therefore, in order to close the set of equations, i.e., to make the number of equations equal to the number of unknowns, the exchange fluxes must be expressed in terms of other resolved variables, namely the state variables relating to the sub-regions between which the mass fluxes are being exchanged - we call this the closure problem. The balance equations must be closed in such a way that the adopted closure relations encapsulate what is presently known about the actual processes and mechanisms governing these fluxes. They will also be expected to incorporate the effects of sub-grid heterogeneities of climate, soils, topography and vegetation, as expressed through a number of exchange coefficients, which will appear as parameters in the adopted closure relations. In this way, the REW approach parameterizes the effects of variabilities occurring at scales smaller than the REW, and explicitly resolves variabilities occurring over scales larger than the REW. An REW together with its closure relations is therefore a functional unit that mimics the hydrological functioning of a larger control volume i.e. a subcatchment in the landscape.

Our approach for developing closure relations builds on the disaggregation-aggregation approach outlined by Sivapalan (1993) and Viney and Sivapalan (2004), and was significantly influenced by the scaleway concept of Vogel and Roth (2003). This approach can provide some guidance towards dealing with multi-scale heterogeneities with given structures, textures, material properties, and appropriate process models, to come up with appropriate closure relations as well effective material properties at the next larger scale. Here, we repeat a few terms for the the sake of clarity. The scale of observation is the linear extent of the entire investigated region (regardless of spacing and support of measurements). Structure is the one that is composed of form elements comparable in size with the scale of observation, while the textural elements are very much smaller. To derive closure relations at the given study area, it is a prerequisite to recognize and represent explicitly different structures, textures, and material properties at the given scale to come up with appropriate closure relations. Depending on different types of saptial heterogeneities across observational scales, the type of structural organization emergent at the larger scale is also different, e.g., macroscopic homogeneity, discrete hierarchy, continuous hierarchy, and fractals as shown by Vogel and Roth (2003). In this light for a standard distributed hydrological model, the soil matrix is the texture and is well described by Richards' equation and appropriate soil hydraulic functions that represent the topology and connectivity of the pore spaces. Structures at this scale may be the spatial patterns of soils, including soil layering and possible preferential pathways. Moving on to the REW scale, it can be expected that the spatial patterns of soils and preferential pathways will affect mass exchange fluxes, and will in turn become the "texture" at the REW scale. In this sense, it is essential to assess REW-scale textural properties and associated parameters, which embed the effects of the sub-scale structures on the mass exchange fluxes at the REW scale. 


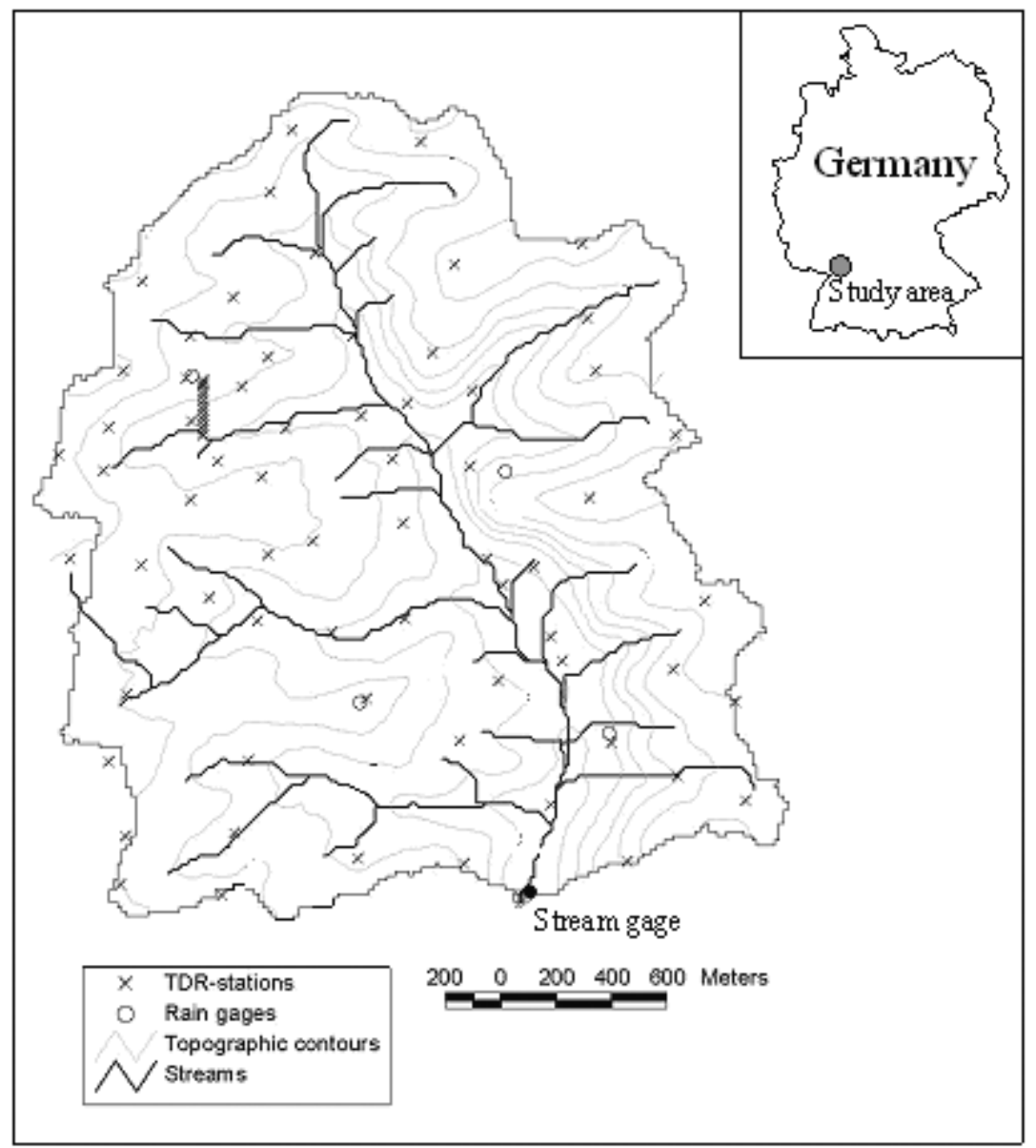

Fig. 2. Observational network of the Weiherbach catchment, after Zehe et al. (2005a): soil moisture was measured at 61 TDR stations at weekly intervals (crosses). Topographic contour interval is 10 [m].

Within this study we will assume that the hydrological micro-scale, i.e. the scale of small experimental catchments, is a key scale for the derivation of physically sound closure relations because:

- the micro-catchment scale is small enough so that we can gain a reasonable understanding of how spatial patterns of soils and preferential pathways affect various mass exchange fluxes through the use of detailed field observations and distributed models; and

- the micro-catchment scale is large enough so that we can set up a model based on the REW-approach to simulate average or typical hydrological dynamics in this region and to perform comparative simulations.

These assumptions provide the main justification for using the micro-scale experimental catchment, Weiherbach, in south-west Germany, for the derivation of the requuired closure relations.

\subsection{Study area}

As mentioned above, Weiherbach catchment was selected as the study area for developing closure relations for various mass exchange fluxes. The Weiherbach is a rural catchment of $3.6\left[\mathrm{~km}^{2}\right]$ size situated in a Loess area in the south-west of Germany. Geologically, it consists of Keuper and Loess layers up to $15[\mathrm{~m}]$ thick. The climate is semi-humid with an average annual precipitation of 750 to 800 [mm/yr], average annual runoff of $150[\mathrm{~mm} / \mathrm{yr}]$ and annual potential evapotranspiration of 775 [mm/yr] (Zehe et al., 2001). More than 95\% of the catchment area is used for cultivation of agricultural crops or pasture, $4 \%$ is forested and $1 \%$ is paved area. Most of the Weiherbach hillslopes exhibit a typical Loess catena with moist but drained Colluvisols located at the foothills, and drier calcareous Regosols located at the hilltops and midslope regions. Figure 2 gives an overview of the observational network of the Weiherbach catchment. Rainfall inputs were measured in a total of 6 rain gages, and streamflows were monitored at a temporal resolution of $6[\mathrm{~min}]$. The catchment area up to the stream gauge is $3.6\left[\mathrm{~km}^{2}\right]$. Soil 
moisture was measured at up to 61 locations at weekly intervals using two-rod TDR equipment that integrates over the upper $15 \mathrm{~cm}$, upper $30 \mathrm{~cm}$, upper $45 \mathrm{~cm}$ and upper $60 \mathrm{~cm}$ of the soil. The soil hydraulic properties of typical Weiherbach soils, after van Genuchten (1980) and Mualem (1976), were measured in the laboratory using undisturbed soil samples taken along transects at several hillslopes, with up to 200 samples per slope (Table 1, Schäfer, 1999).

The Weiherbach catchment offers considerable advantages for the development of closure relations. Firstly, it has been maintained as a significant experimental catchment over the past many years. There is a wealth of information regarding the geology and soil properties, and field experimentation has generated a wealth of measurements of various water fluxes and internal soil state variables, such as soil moisture and tracer concentrations. Secondly, as mentioned before, a detailed physically based model CATFLOW (using the finite difference scheme) (Maurer, 1997; Zehe et al., 2001, Zehe and Blöschl, 2004), has been developed and successfully verified in this catchment. Zehe et al. (2001) showed that in this catchment, which is situated in a Loess area in Germany, a model structure which only incorporates typical (not actual) spatial patterns of soils, vegetation and preferential pathways in that loess landscape, and neglects local scale statistical variability, is sufficient to explain a large part of the observed variability of hydrological processes at the catchment scale. Within a 1.5 year long simulation the catchment model predicted catchment scale runoff response with a Nash-Sutcliffe efficiency of 0.82 , explained more than $92 \%$ of the evapo-transpiration observed at a meteorological station and yielded at the same time reasonable predictions of soil moisture time series observed at 61 locations in the catchment with correlation coefficients ranging between 0.65 and 0.75 (Zehe et al., 2001; Zehe et al., 2005a). In this study we will employ this "landscape and process compatible" model structure for the development of the closure relations relating to seepage (subsurface) outflow from simulated hillslope scale drainage experiments, and the pressuresaturation and conductivity-saturation relationships.

\section{Derivation of closure relations and the CREW model}

\subsection{Different approaches for assessing closure relations}

In this section, we review the upscaling methods that are currently available to develop closure relations for mass exchange fluxes. We classify these upscaling methods into four categories: field experiments, theoretical/analytical derivations, numerical experiments, and hybrid approaches.

\subsubsection{Assessing closure relations based on field experi- ments}

The field experimental approach seeks to find closure relations from the analysis of data obtained in the field, either in a routine manner or through focused intensive field experiments. Empirical closure relations based on field observations may be the best candidates for the REW scale closure relations, because they best represent the intrinsic natural variability occurring within the study catchment. These include nonlinear and threshold behavior commonly exhibited in many catchments, which are hard to represent using current small-scale theories. Unfortunately, in most cases, field monitoring of catchments is limited to rainfall, runoff, and potential evaporation, which are not sufficient to derive closure relations. At the minimum, the development of closure relations requires measurements of internal state variables in different sub-regions of the catchment system. Unfortunately, currently there are no measurement techniques available that allow observations of internal states and subsurface structures for scales larger than the plot- or small field scale (Schulz et al., 2006). While they have been monitored as part of some focused field experiments around the world, such as soil moisture measurement at the Tarrawarra catchment in southern Victoria, Australia (Western and Grayson, 1998), and the Weiherbach catchment, and at over 600 stations from around the globe (Robock et al., 2000), mostly by employing a distributed network of point measurements, the corresponding data from these field experiments alone is not sufficient for derivation of closure relations. The work of Duffy (1996) at the Shale Hills catchment in central Pennsylvania is an exception to this trend, and showed that closure relations, notably the storage-discharge relationship relating to shallow subsurface flow, can be derived on the basis of carefully conducted field experiments, in combination with numerical modelling.

\subsubsection{Analytical approach to the derivation of closure rela- tions}

In the theoretical/analytical approach, the emphasis is on deriving closure relations through analytical integration or upscaling of small-scale physically based equations through mathematical manipulation. A widely used approach is the derivation of effective parameters by means of coarse graining, as suggested by Dagan (1989), Attinger (2003) and Lunati et al. (2002), which is based on assumptions on the probability distributions of key parameters, e.g. the hydraulic conductivity. While this is a useful approach for groundwater, it is too simple for the unsaturated zone and surface processes. This is firstly because the variability of these processes is controlled by the nonlinear interaction of several structures/patterns in a catchment e.g. vegetation, soil hydraulic properties, macroporosity and topography, where the state of the catchment determines which of these patterns is the dominant one (Zehe and Blöschl, 2004). And secondly, the spatial characteristics of these patterns, i.e. the correlation structure and more importantly their connectivity may not be captured with simple analytical functions (Blöschl and Zehe, 2005). However, in some cases this approach has the advan- 
tage that the resulting closure relations, as well as the consequent REW scale parameters, retain some or most of their traditional meaning, and therefore there is a chance that they can be estimated by referring back to a mapping of landscape and/or climatic properties.

\subsubsection{Numerical simulation approach to the derivation of closure relations}

The numerical simulation approach seeks to derive closure relations based on the comprehensive simulated datasets that can be generated through the application of detailed, distributed physically-based hydrological models that are based on small-scale physical theories, under well defined boundary conditions. In contrast to the analytical approach, these models may account explicitly for all the patterns of vegetation, soil properties, macropores and topography, and their nonlinear interactions, that may be controlling the surface and subsurface flows. Closure relations maybe derived from numerical model output by averaging the state variables and parameter fields to the catchment or REW scale and postulating parametric relations. However, the main problem with this approach is that the patterns of vegetation, soil properties (soil hydraulic functions) and macropores are generally unknown. Therefore, one has to make reasonable assumptions about the heterogeneities to be expected in the study catchments. For example, Binley et al. (1989a, b) employed a fully three dimensional model based on the Richards equation, coupled with a simple linear routing approach, for deriving hillslope scale effective hydraulic conductivities for stochastically generated heterogeneous parameter fields. This worked well for weakly heterogeneous systems of high average hydraulic conductivities ranging from 0.05 to $0.2 \mathrm{~cm} / \mathrm{min}$. In the alternative, one can work in well instrumented research catchments to develop the closure relations, as we do in the present study. In such cases the numerical simulation approach can nevertheless be a good starting point for developing closure relations at the catchment scale that are accurate to first order (Zehe et al., 2005a; Kees et al., 2002; Kees et al., 2004). Zehe et al. (2005a) and Kees et al. (2004) presented examples of the development of closure relations for a hillslope scale water balance model with a transient numerical solution of continuum-scale model.

\subsubsection{Hybrid approaches for assessing closure relations}

In the present study we also follow the hybrid approach, which is a combination of any of the above methods presented above. Viney and Sivapalan (2004), following Robinson and Sivapalan (1995), derived closure relations for catchment scale infiltration capacity as a function of the cumulative volume of infiltrated water on the basis on numerical experiments and catchment response data. They tested the effects of different storms as well as different topogra- phies on this relationship, and through these sensitivity analyses parameterized the relationship in terms of storm duration, storm depth, temporal pattern and catchment topography. This led to an acceptable empirical closure relation for infiltration rate that could be embedded within a large-scale catchment model.

In a similar way, we apply a number of these methods to develop closure relations for crucial mass exchange fluxes appearing in the REW scale balance equations; these derivations are presented in Sects. 3.2.1 to 3.2.6. Supplementary parametrizations such as a geometric relationship for saturated surface area, the REW scale water-retention curve, and the hydraulic conductivity versus saturation relationship, are derived in Sects. 3.3.1 to 3.3.3. In Sect. 3.4 the developed closure relations are combined, with the original balance equations, yielding a set of equations which form the basis of the CREW model.

\subsection{Closure relations for mass exchange fluxes}

\subsubsection{Infiltration $e^{u c}$}

For the infiltration process during rainfall events we directly use the results of Rogers (1992) who developed an areal average infiltration capacity model based on the standard GreenAmpt equation. He assumed that only saturated hydraulic conductivity is spatially variable, and that it follows a lognormal distribution. All other soil parameters were assumed constant, with the justification that saturated hydraulic conductivity is much more variable than the other parameters and has a greater impact on infiltration (Bresler and Dagan, 1983). The resulting infiltration capacity equation has the following form:

$\overline{f^{*}}=\overline{K_{s}}\left[1+\alpha^{u c} \frac{\left|\Psi_{f}\right|\left(\theta_{s}-\theta_{i}\right)}{\bar{F}}\right]$

where $\overline{f^{*}}$ is spatially averaged infiltration capacity, $\overline{K_{s}}$ is mean saturated hydraulic conductivity, $\left|\Psi_{f}\right|$ is soil's matric potential head at the wetting front, $\theta_{s}$ is saturated soil moisture content, $\theta_{i}$ is initial soil moisture content, $\bar{F}$ is spatially averaged cumulative volume of infiltration and $\alpha^{u c}$ is a "scaling" parameter related to the variability of hydraulic conductivity. To adapt Eq. (12) within the REW modeling framework, we need to find a match between state variables in Eq. (12) and those of the REW approach. Since $\bar{F}$ corresponds to infiltrated water depth into the unsaturated zone, it was replaced by $s^{u} y^{u}$, where $s^{u}$ is degree of saturation in the unsaturated zone and $y^{u}$ is average thickness of the unsaturated zone along the vertical. $\left|\Psi_{f}\right|$ and $\theta_{s}-\theta$ are replaced by $|\Psi|$ and $\left(1-s^{u}\right) \varepsilon^{u}$ respectively, where $|\Psi|$ is the soil's matric potential head (which is a function of saturation degree in the unsaturated zone) and $\varepsilon^{u}$ is soil porosity in the unsat- 
urated zone. The resulting form of the infiltration capacity equation is:

$\overline{f^{*}}=\overline{K_{s}}\left[1+\alpha^{u c} \frac{|\Psi|\left(1-s^{u}\right) \varepsilon^{u}}{s^{u} y^{u}}\right]$

where $\alpha^{u c}$ embeds within it the effects of not only the spatial variability of soils, but also of the space-time variability of the wetting front position during the infiltration process. It is clear, that this key parameter is difficult to estimate a priori and further work is required in different catchments to get at this number. The infiltration capacity Eq. (13), which is based on and resembles the standard Green-Ampt infiltration equation, still has much room for improvement to account for new findings from field experiments and to provide improved predictions of ponding time, and the effect of rainfall heterogeneities on the infiltration process.

Finally, as in Reggiani et al. (2000), the actual infiltration flux $\left(e^{u c}\right)$ during rainfall events can be expressed as:

$e^{u c}=\min \left[i \omega^{u}, \overline{f^{*}} \omega^{u}\right]$

where $i$ is rainfall intensity and $\omega^{u}$ is the surface area fraction occupied by the unsaturated zone.

\subsubsection{Bare soil evaporation and transpiration by root uptake}

Closure relations for bare soil evaporation and transpiration by root uptake were derived analytically based on the exfiltration capacity model of Eagleson (1978b,c), assuming that the soil hydraulic conductivity is spatially variable and follows a log-normal distribution. The resulting closure relations have the following final form:

$e_{w g}^{u}=\min \left[\left(e_{p}+M k_{v} \overline{e_{p}}\right) \omega^{u}, \overline{f_{E T}^{*}} \omega^{u}\right]$

$\overline{f_{E T}^{*}}=\alpha_{w g}^{u} \frac{\overline{K_{s}}}{\left(1-s^{u}\right) y^{u}} \frac{\left(s^{u}\right)^{2+d} \varepsilon^{u}\left|\Psi_{b}\right|}{m}$

where $e_{p}$ is potential evaporation rate from the bare soil surface, $M$ is the vegetated fraction of land surface, i.e., canopy density, $k_{v}$ is the ratio of potential rates of transpiration and soil surface evaporation, $\overline{e_{p}}$ is the long-term (time averaged) rate of potential (soil surface) evaporation, $\overline{f_{E T}^{*}}$ is the spatially averaged combined exfiltration capacity due to bare soil evaporation and transpiration by root uptake, $m$ is pore size distribution index, $c$ is pore disconnectedness index, $\varepsilon^{u}$ is soil porosity in the unsaturated zone, $d$ is diffusivity index, $\left|\Psi_{b}\right|$ is the bubbling pressure head, and $\alpha_{w g}^{u}$ is a parameter related to variability of saturated hydraulic conductivity and exfiltration diffusivity. The full derivation of these equations and the meaning of $\alpha_{w g}^{u}$ is presented in detail in Appendix A.

Hydrol. Earth Syst. Sci., 11, 819-849, 2007

\subsubsection{Groundwater recharge/capillary rise}

Groundwater recharge/capillary rise, in general, refers to the mass exchanges between the unsaturated zone and the saturated zone that lies below it. Depending on its dominant direction it can take on different meanings. It will be called net recharge if the net water flow is vertically downward into the saturated zone, while it will be termed capillary rise if the net flow is vertically upward into the unsaturated zone. The direction of flow at any time is governed by the status of momentum balance within the unsaturated zone, expressed through the resulting unsaturated zone vertical velocity $v_{z}^{u}$. In this paper, as a first step, we develop somewhat simple closure relationship for the recharge flux/capillary rise of the following form:

$e^{u s}=\alpha^{u s} \omega^{u} v_{z}^{u}$

where $\alpha^{u s}$ is considered as a constant of proportionality linking the average vertical velocity with the entire unsaturated zone and the recharge/capillary rise at the bottom, which is a boundary flux. The form of this closure relation, Eq. (17), has a similar form to that of Reggiani et al. (2000). Here it should be pointed out that the improvement of this closure relation lies not only in the way of parameterizing $e^{u s}$ but also in the way of relating $v_{z}^{u}$ to both relevant state variables, material properties and capillarity by adopting appropriate expressions for the non-equilibrium part of the momentum exchange terms (compare Eq. 34). At the moment, we are adopting first-order Taylor series expansion for deriving $v_{z}^{u}$ which leads to the result that appears in Eq. (8) and similar to the equation adopted by Reggiani et al. (2000).

\subsubsection{Saturated and concentrated overland flow}

A closure relation for saturated overland flow was obtained by adopting the numerical simulation approach, and using the steady-state solution for the kinematic wave equation governing overland flow, improved to consider the effect of field capacity on water flow through soil media (Ichikawa and Shiiba, 2002). Based on the results of numerical simulations applied to the Weiherbach catchment, several functional relationships between saturated overland flow and the state variables relating to overland flow were explored. It was found from these simulations that $e^{r o}$ is linearly proportional to the product of the average flow depth of the saturated overland flow zone $\left(y^{o}\right)$ and the average velocity of overland flow $\left(v^{o}\right)$, i.e., $e^{r o}=\alpha_{1}^{r o} y^{o} v^{o}$, where $\alpha_{1}^{r o}$ is an exchange coefficient that remained to be estimated. A further investigation was carried out to examine the dependence of $\alpha_{1}^{r o}$ on the total length of channels within the REW. For this investigation, the Weiherbach catchment was divided into 39 basin groups, and these were subjected to rainfall events with intensities ranging from 0.1 to 40 [mm/hr]. Figure 3 presents the results describing the dependence of $\alpha_{1}^{r o}$ on total hillslope width as a measure of total channel length. The relationship between 


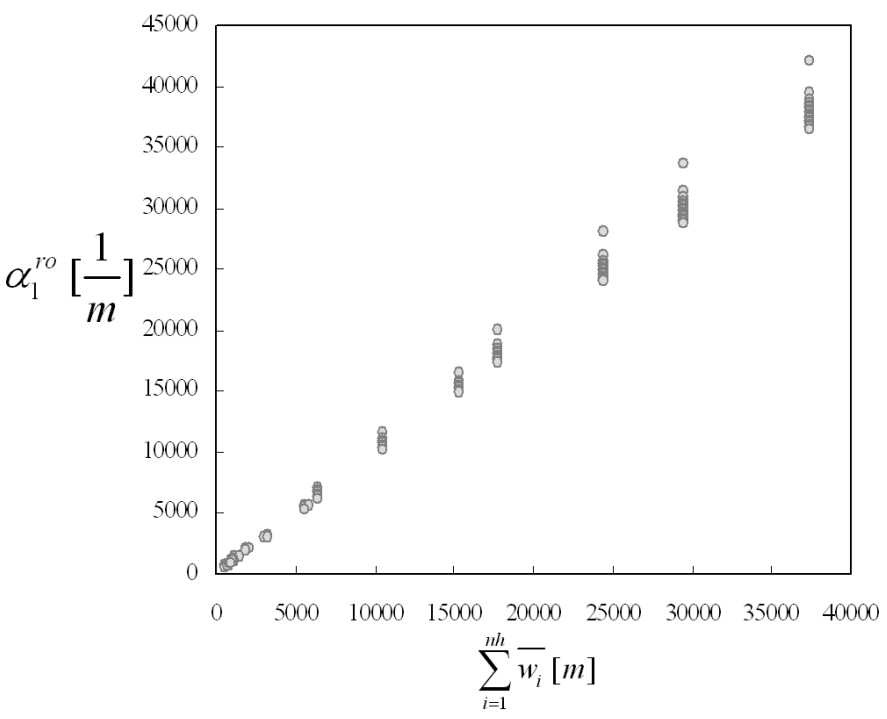

Fig. 3. Dependence test of parameter $\alpha_{1}^{r o}$ to hillslope width.

$\alpha_{1}^{r o}$ and hillslope width does show a small dependence on rainfall intensity, as expressed through the scatter, which increases as the size of the REW increases. Generally, however, a strong linear dependence on total hillslope width can be seen in Fig. 3. On the basis of these results, the following closure relation is adopted for saturated overland flow:

$e^{r o}=\alpha^{r o} \xi^{r} y^{o} v^{o}$

where $\xi^{r}$ is defined as the length of channels per unit surface area, which can be considered as equivalent to the catchment's drainage density, and $\alpha^{r o}$ is now a dimensionless constant of proportionality. For the Weiherbach catchment, $\alpha^{r o}$ is 1.0 from Fig. 3.

It is assumed that overland flow over the concentrated overland flow zone, generated by the infiltration excess mechanism, could be closed with a function of the same form as that of saturated overland flow. Therefore, the suggested closure relation is:

$e^{o c}=\alpha^{o c} \xi^{r} y^{c} v^{c}$

where $\xi^{r}$ is the length of the channel reach per unit surface area, or drainage density. In both closure relations for saturated and concentrated overlad flow, we should take more care about the way to describe velocity terms, $v^{c}$ and $v^{o}$, in such a way that the velocities estimated from Eqs. (9) and (10), with carefully chosen surface roughnesses and surface slopes at the REW scale, should be compatible with the corresponding averages estimated from predictions of the distributed model.

\subsubsection{Seepage outflow}

The closure relation for seepage outflow was obtained by using the numerical simulation approach. For this the phys- ically based distributed model CATFLOW (Maurer, 1997; Zehe et al., 2001) was used, and applied to the Weiherbach catchment in Germany. CATFLOW is capable of simulating continuous space-time dynamics of water flow and transport of solutes in the upper soil layer of small rural catchments, including channel network and vegetation cover, on event and seasonal time scales, over the three dimensional spatial domain. CATFLOW utilizes the 2-D Richards equation to model water the soil, Penman-Monteith equation for evapotranspiration, and 1-D Saint-Venant equation for runoff from hillslopes, as well as flow in the drainage network. CATFLOW is also capable of handling the presence of macropores (Zehe et al., 2001), with a simplified effective parameter approach. If water saturation in macroporous soil exceeds field capacity, the bulk hydraulic conductivity at this point is assumed to increase linearly with saturation up to a maximum value at full saturation of the soil. This maximum value is determined by the macroporosity factor $f_{M}$. The macroporosity factor is the ratio of the water flow rate in the macropores, in a model element of area A, with the saturated water flow rate in the soil matrix. This is a simplified approach, that is however suitable to represent the effect of enhance infiltration due to macropore flow at grid scale of order 1000-10000 $\mathrm{m}^{2}$, as shown by Zehe et al. (2001, 2005b). Because CATFLOW has been verified on the Weiherbach catchment using tracers and rainfall-runoff data, with good success, we are confident that the model is able to reproduce well the internal mechanisms of water movement, storage, runoff generation and evapotranspiration, including the substantial space-time variability.

As a first step, a hillslope with soil catena and a spatial pattern of macroporosity typical for the Weiherbach catchment was chosen as the spatial domain (Zehe and Blöschl, 2004). 
Table 1. Laboratory measurements of average hydraulic properties for typical Weiherbach soils. Definition of parameters after van Genuchten (1980) and Mualem (1976). Saturated hydraulic conductivity $K_{s}$, porosity $\varepsilon$, residual water content $\theta_{r}$, air entry value $\bar{\alpha}$, shape parameter $n$.

\begin{tabular}{lccccc}
\hline & $K_{s}\left[\mathrm{~m} \mathrm{~s}^{-1}\right]$ & $\varepsilon\left[\mathrm{m}^{3} \mathrm{~m}^{-3}\right]$ & $\theta_{r}\left[\mathrm{~m}^{3} \mathrm{~m}^{-3}\right]$ & $\bar{\alpha}\left[\mathrm{m}^{-1}\right]$ & $n[-]$ \\
\hline Calcareous regosol & $2.1 \times 10^{-6}$ & 0.44 & 0.06 & 0.40 & 2.06 \\
Colluvisol & $6.1 \times 10^{-6}$ & 0.40 & 0.04 & 1.90 & 1.25 \\
\hline
\end{tabular}

The hillslope was discretized into 21 nodes horizontally and 21 layers vertically. In the upper $80 \%$ of the hillslope a Loess soil is located; the lower $20 \%$ consist of Colluviosol. The dependence of soil water potential and unsaturated hydraulic conductivity on soil moisture saturation is expressed in terms of the van Genuchten (1980) and Mualem (1976) formulations (Table 1) given below:

$$
\begin{aligned}
& s=\frac{\theta-\theta_{r}}{\theta_{s}-\theta_{r}}=\left[\frac{1}{1+(\bar{\alpha}|\psi|)^{n}}\right]^{\frac{n-1}{n}} \\
& K=K_{s} s^{\frac{1}{2}}\left[1-\left[1-s^{\frac{n}{n-1}}\right]^{\frac{n-1}{n}}\right]^{2}
\end{aligned}
$$

Vertical soil depth is set at $2[\mathrm{~m}]$. Following the suggestions of Zehe et al. (2001) a spatially variable macro-porosity factor was assigned along the length of the hillslope profile, with fixed relative portions $0.6 f_{M}$ for the upper $70 \%$, $1.1 f_{M}$ for the 70 to $85 \%$, and $1.5 f_{M}$ for the $85 \%$ to $100 \%$ part of the hillslope. The average macro-porosity factor was taken to be $f_{M}=2.1$. Zehe et al. (2001) showed that a catchment model consisting of 169 of these typical hillslopes and the related drainage network is sufficient to yield good predictions of discharge (Nash-Sutcliffe efficiency of 0.82 ), observed ET (correlation 0.92 without a bias) and observed soil moisture dynamics at 61 TDR stations (correlations between 0.65-0.75). This hillslope can therefore be deemed as typical heterogeneity or "structure" in the Weiherbach catchment, whose effects have to be parameterised in the form of texture at the REW scale. To check additionally the possible influence of small scale variability of saturated hydraulic conductivity and porosity, we added a local fluctuation (in form of multipliers) around the average values presented in Table 1, which was generated using the Turning Bands algorithm using two variograms (nugget variogram with sill $=0.023$; spherical variogram with range $=2 \mathrm{~m}$, and sill $=0.047$, average was of course 1 ) to generate spatially correlated random fields.

For this typical hillslope a series of simulations were carried out with two different specified boundary conditions. In the first case, the soil is initially fully saturated with or without rainfall events across the hillslope. In this case drainage is occurring over the entire spatial domain of the hillslope, and will reach a steady state after sufficiently long simulation time; this is named the drainage experiment. In the second case, the soil is initially dry and rainfall occurs continuously at a constant rate, and infiltration is the main hydrological process; this is called the infiltration experiment. The boundary conditions used and the mass exchange fluxes required for these studies are described in Fig. 4a. A zero flux boundary condition is assigned to the lower and left boundaries of the slope, as shown in Fig. 4a.

The applied artificial rainfall range is from 0 to $1.0[\mathrm{~mm} / \mathrm{hr}]$ in steps of $0.1[\mathrm{~mm} / \mathrm{hr}]$, and $10,20,30$ and $40[\mathrm{~mm} / \mathrm{hr}]$, which were chosen based on the experience of previous numerical experiments and data interpretation. At rainfall intensities less than $1.0[\mathrm{~mm} / \mathrm{hr}]$, the hillslope becomes almost fully saturated after a 20-month simulation period from 21/04/1994 to 31/12/1995; this could be regarded as steady state. It is believed that the most transient solution affecting water dynamics in the Weiherbach catchment could be obtained through these simulations. Based on the analysis of the results from infiltration and drainage experiments, many functional relationships between seepage flow and combinations of state variables were tested. The results are presented in Fig. 4b; while the empirical results do display certain multi-valuedness, the general trend is still suggestive of a power law relation. Therefore, as a first step, an empirical closure relation for seepage flow of the following power law form is adopted:

$e^{o s}=\alpha^{o s}\left[\frac{S}{|\Psi|}\right]^{\alpha_{3}^{o s}}$

where $S$ is the degree of saturation estimated over the entire volume of soil (including both the unsaturated and saturated zones), and $|\Psi|$ is the average matric potential head of the soil over the entire unsaturated zone. Both are different from the corresponding point scale values used in Eq. (20) (compare Table 1), and $\alpha^{o s}$ and $\alpha_{3}^{o s}$ are parameters that remain to be estimated. However, the quotient of saturation and matric potential, has an interesting analog at the REV scale, where the derivative of the $\partial \theta / \partial \Psi$ is known as water capacity of a porous medium. The quotient at the REW scale has a similar meaning, where seepage flow is determined by the water capacity of the REW scale porous medium. This is very plausible. The issue of possible multi-valuedness of the two parameters involved is an important problem but is left for future research within the context of an uncertainty assessment of CREW. 


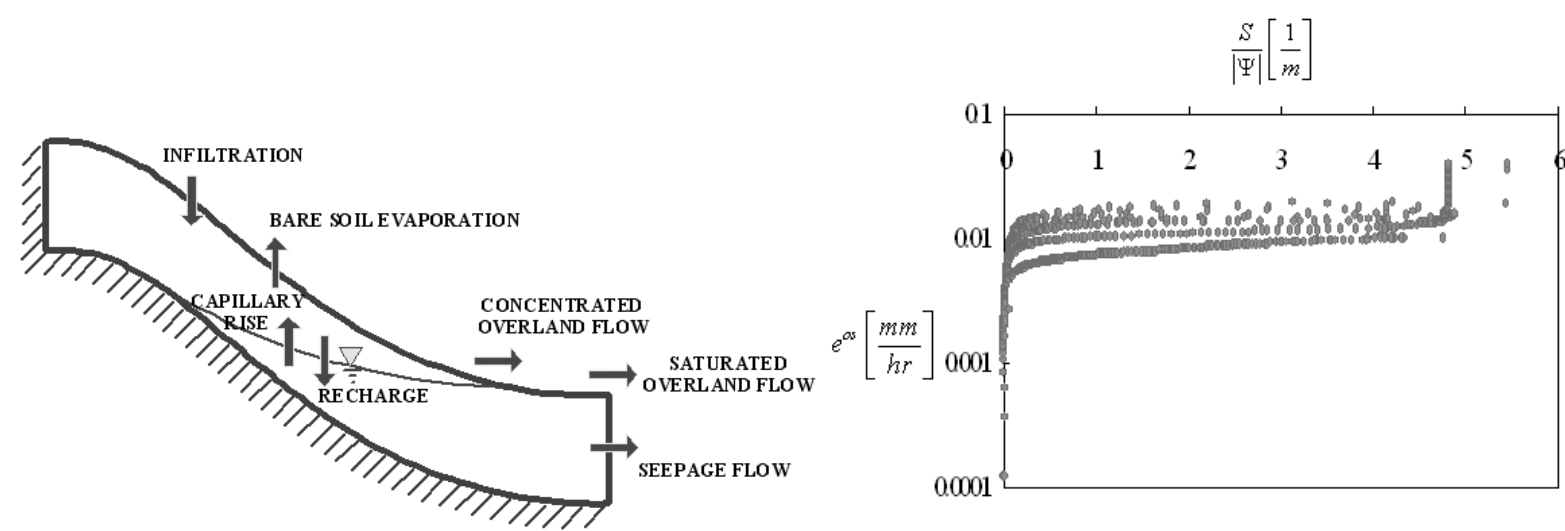

(a)

(b)

Fig. 4. (a) Hillslope setting for developing closure relation for seepage flux, using CATFLOW simulation; and (b) the best candidate for the seepage flux closure relation where $e^{o s}, \mathrm{~S}$, and $|\Psi|$ are seepage outflow, saturation degree over the entire volume of soil, and the average matric potential head of the soil over the entire unsaturated zone.

Subsequent tests of parameter dependence revealed a relationship of the form $\alpha^{o s}=\alpha_{1}^{o s}{\overline{K_{S}}}^{\alpha_{2}^{o s}}$, while $S$ is replaced by the ratio of stored water depth in the soil at given time step, $y^{u} s^{u} \omega^{u}+y^{s}$, to the total soil depth, Z. Accordingly, the closure relation for seepage outflow can be written as:

$e^{o s}=\alpha_{1}^{o s}{\overline{K_{s}}}_{2}^{\alpha_{2}^{o s}}\left[\frac{y^{u} s^{u} \omega^{u}+y^{s}}{Z|\Psi|}\right]^{\alpha_{3}^{o s}}$

The closure relation for seepage flux, or Eq. (23), was developed for the hillslope setting of Fig. 4a, in which seepage flux is only allowed at the right hand side of the slope. Equation (23) must therefore be modified to incorporate the effect of rising water table causing an increase of the seepage face. For this reason, following Sloan and Moore (1984), Eq. (23) is modified to account for water table rise, and this leads to:

$e^{o s}=\omega^{o} \alpha_{1}^{o s}{\overline{K_{s}}}_{2}^{\alpha_{2}^{o s}}\left[\frac{y^{u} s^{u} \omega^{u}+y^{s}}{Z|\Psi|}\right]^{\alpha_{3}^{o s}}$

For the Weiherbach catchment, estimated values for $\alpha_{1}^{o s}, \alpha_{2}^{o s}$, $\alpha_{3}^{o s}$ are $0.01,0.60$, and 0.31 respectively.

\subsubsection{Channel flow, and remaining closure relations}

For the channel inflow and outflow sections, the following closure relation is suggested based on continuity considerations:

$\sum_{l} e_{l}^{r A}+e_{\text {ext }}^{r A}=\sum_{l} \frac{m_{l}^{r} v_{l}^{r}}{\Sigma}-\frac{m^{r} v^{r}}{\Sigma}$

where $\sum_{l} e_{l}^{r A}$ are inflow or outflow discharge thorough channel stream network at the inlet or outlet of each REW and $e_{e x t}^{r A}$ is outflow discharge at the outlet of the whole catchment system. $m_{l}^{r}$ and $m^{r}$ are channel cross sectional area of the $l$ th neighbouring REW and the REW of interest respectively. $v_{l}^{r}$ and $v^{r}$ are streamflow velocities in channel network at the $l$ th neighbouring REW and the REW of interest respectively. $\Sigma$ is the projected surface area of the REW of interest onto the horizontal plane.

A small steady groundwater flow is allowed to maintain a minimum water quantity in channel during dry periods, and a zero flux condition is assigned across the REW mantle segment, which is a part of the REW system boundary delimiting the spatial extent of a REW laterally in the subsurface zone. Rainfall or evaporation to and from the c-, o- and r-zones are assumed to be directly proportional to rainfall intensity or potential evaporation multiplied by the area fraction of each zone. All of the closure relations developed above are summarized in Table 2, and compared against the closure relations previously proposed by Reggiani et al. $(1999,2000)$ on intuitive grounds.

\subsection{Constitutive relations for the Weiherbach catchment}

\subsubsection{Geometric relationship for saturated surface area}

It is very important to predict the saturated surface area fraction that responds to saturated zone depth in reasonable way, since the saturated surface area is directly related to the generation of saturation excess overland flow, as well as seepage outflow. Also, as indicated above, some of the adopted closure relations require the estimate of saturated surface area.

Saturated surface area is the fraction of catchment area caused by the intersection of the water table with the land surface. Hence, it is governed by the dynamics of saturated zone thickness, and by surface topography. Therefore, the relationship between saturated surface area and the depth of saturated zone can be obtained by an understanding of topographic control on saturation area dynamics. In this study, we 
Table 2. Closure relations for exchanging mass flux.

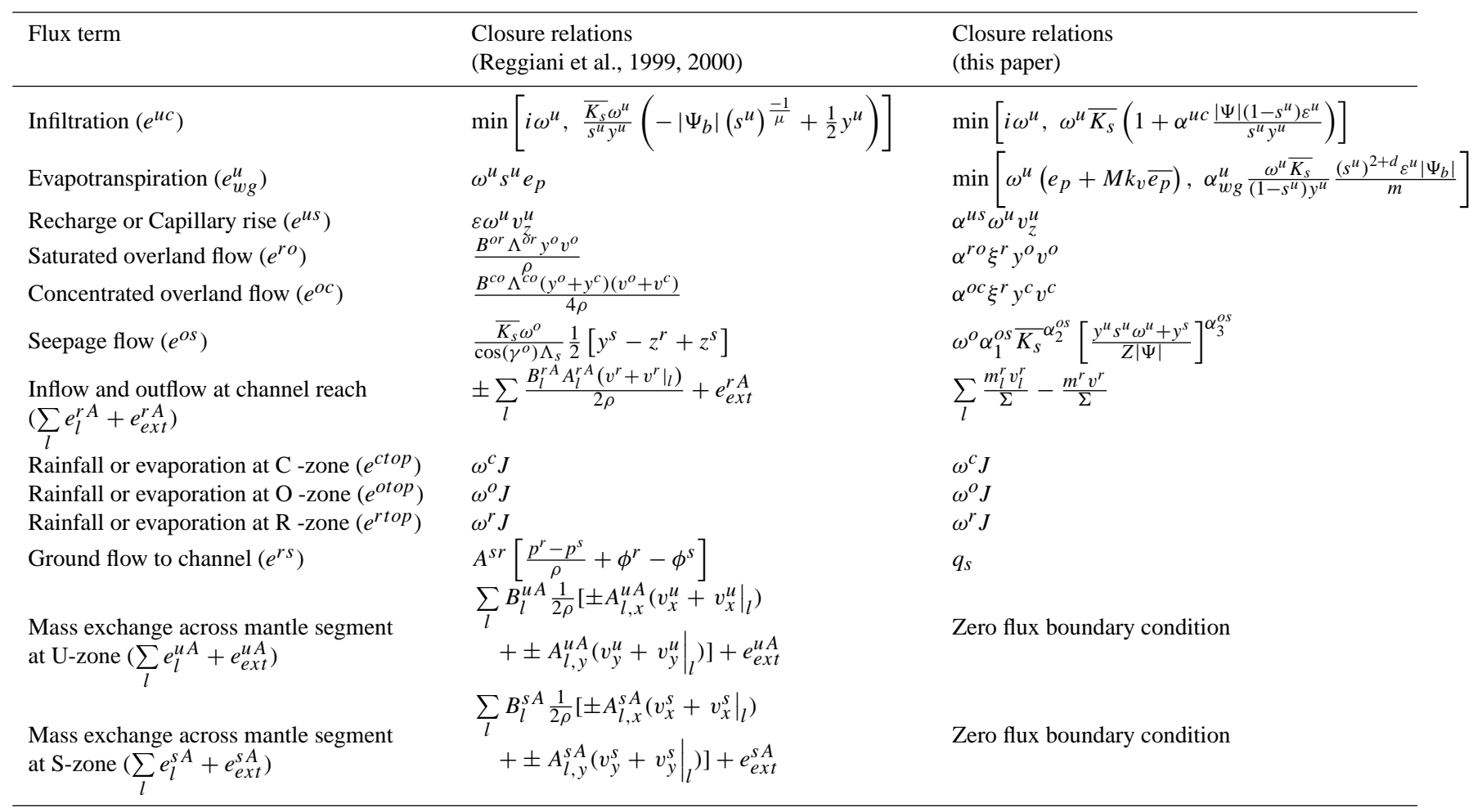

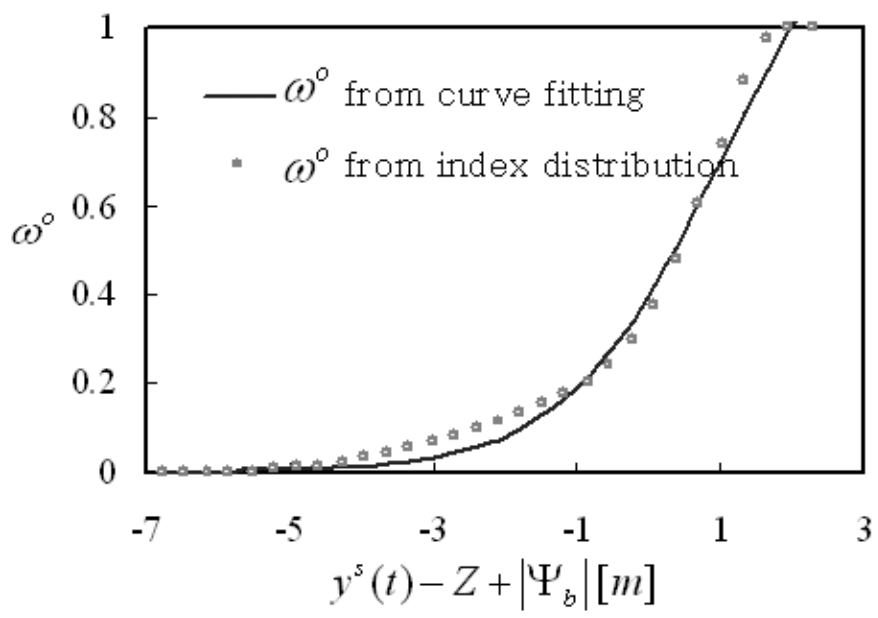

Fig. 5. Geometric relationship for saturated area fraction as a function of averaged thickness of saturated zone.

investigated a possible functional relationship between saturation zone depth and saturated surface area by means of the topographic wetness index of TOPMODEL (Beven and Kirkby, 1979).

Application of TOPMODEL theory to the Weiherbach catchment produced the functional form, shown in Fig. 5, for the geometric relationship between saturated surface area fraction and depth of the saturated zone. This relationship can be expressed as:

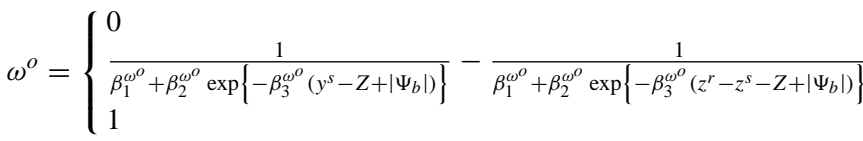

$$
\begin{aligned}
& \text { if if } y^{s} \leq z^{r}-z^{s}
\end{aligned}
$$




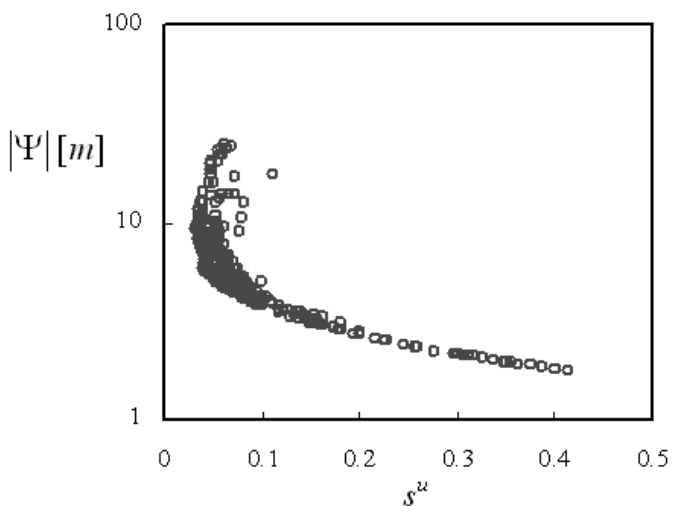

(a)

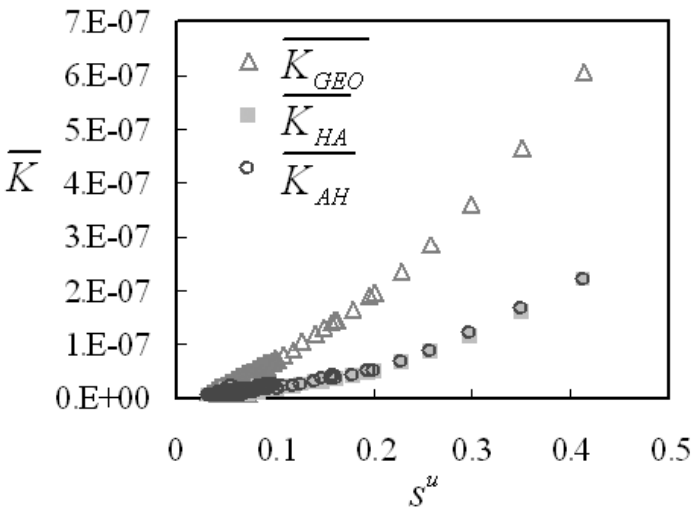

(b)

Fig. 6. Catchment scale (a) water retention curve, and (b) hydraulic conductivity curve, based on CATFLOW simulations.

$$
\begin{aligned}
& \text { if if } z^{r}-z^{s}<y^{s}<Z \\
& \text { if } y^{s}=Z
\end{aligned}
$$

where $z^{r}, z^{s}$ and $Z$ are average elevation of channel bed from datum, average elevation of the bottom end of the REW above datum, and the average thickness of the subsurface zone respectively, and $\beta_{1}^{\omega^{o}}, \beta_{2}^{\omega^{o}}$ and $\beta_{3}^{\omega^{o}}$ are parameters to be estimated. The details of the derivation procedure for the geometric relationship for saturated surface area are shown in Lee et al. (2005b). For the Weiherbach catchment, $\beta_{1}^{\omega^{o}}=0.71$, $\beta_{2}^{\omega^{o}}=1.79, \beta_{3}^{\omega^{o}}=0.92$ from Fig. 5 .

\subsubsection{REW scale water retention curve: capillary pressure} vs saturation relationship

The REW scale water retention curve represents the soil's matric potential head in the unsaturated zone as a function of the saturation degree. Such a relation is needed to describe capillary rise of groundwater but also to estimate rate of infiltration (see Eqs. 13 and 34). In the closely related study of Zehe et al. (2006) numerical experiments were performed to derive this REW scale water retention curve based on time series of both soil saturation and matric potential, that have been integrated over the simulation domain. In this study we use the full size distributed catchment model with the typical structures defined by Zehe et al. (2001) as well as observed boundary conditions (athmosphere at the top, free drainage at the bottom) and simulated the one year period 21/04/1994 to $31 / 12 / 1995$. The idea is to link the volume integrated saturation value and the REW scale matric potential that has evolved under natural conditions (Fig. 6a) by the following functional relationship:

$$
|\Psi|=\beta_{1}^{|\Psi|}\left\{s^{u}\right\}^{-\beta_{2}^{|\Psi|}}
$$

where $\beta_{1}^{|\Psi|}$ is the bubling pressure and $\beta_{2}^{|\Psi|}$ the pore size distribution index. For the Weiherbach catchment,
$\beta_{1}^{|\Psi|}=0.97(\mathrm{~m}), \beta_{2}^{|\Psi|}=0.64$ from Fig. 6a. Zehe et al. (2006) worked at the scale of a typical hillslope and imposed artificial boundary conditions during separate wetting and drainage simulation experiments and investigated the effect of matrix and macropore heterogeneities in this context. They obtained different values for the same relationship (cf. discussion section).

\subsubsection{Hydraulic conductivity vs saturation relationship}

Similarly, the unsaturated hydraulic conductivity is needed to describe the change of hydraulic conductivity as a function of saturation degree, which is in our case only important for recharge and capillary rise (infiltration works with the average saturated hydraulic conductivity according to Eq. (13)). In the light of the scaleway concept (Vogel and Roth, 2003) it is clear that, in general, this relationship will be strongly affected by subscale structures such as macropores. However, the macropore system in the Weiherbach catchment is shallow and does not penetrate into the saturated zone, thus recharge is controlled by soil matrix properties. Furthermore, soils are weakly heterogeneous with respect to their matrix properties (Zehe and Blöschl, 2004). Again we employed two approaches to assess the relationship between unsaturated hydraulic conductivity and saturation degree based on numerical experiments. In this and the approach of Zehe et al. (2006) we use the same parameteric relation:

$\bar{K}=\overline{K_{s}}\left\{s^{u}\right\}^{\beta_{2}^{\bar{K}}}$

where $\overline{K_{S}}$ is the saturated hydraulic conductivity at the REW scale and $\beta_{2}^{\bar{K}}$ is an exponent to account for capillarity that remains to be estimated. In this approach we again use the full catchment scale model and derived $\bar{K}$ by three methods of volume weighted averaging of the REV scale values at the nodes at each time step. The first method is geometric averaging, with the resulting average being denoted as 
$\overline{K_{G E O}}$. The second is harmonic averaging for soil layers on each hillslope, followed by arithmetic average for parallel combinations, leading to an average denoted as $\left(\overline{K_{H A}}\right)$. The third method is the same as the second, except that the steps are reversed, i.e., arithmetic first followed by the harmonic for the layers $\left(\overline{K_{A H}}\right)$. The results of these calculations are presented in Fig. 6b expressented in terms of Eq. (28), with the second parameter being estimated by curve fitting. From Fig. 6b, the estimated $\overline{K_{S}}$ and $\beta_{2}^{\bar{K}}$ values for the Weiherbach catchment are, respectively, $3.0 \times 10^{-6}$, and 1.68 for $\overline{K_{G E O}}$, $8.0 \times 10^{-7}$, and 1.63 for $\overline{K_{H A}}$, and $7.0 \times 10^{-7}$, and 1.49 for $\overline{K_{A H}}$.

In the companion approach Zehe et al. (2006) used the typical hillslope to simulated drainage and wetting experiments again for well defined artificial instead of observed boundary conditions. Regardless of the hydraulic conductivity values that evolved inside the domain, they used the averaged in/outflow at the lower boundary of the domain and the aver- age saturation and the expression for the REW-scale recharge velocity used here (Eq. 34) to derive parameters for Eq. (28). Again they obtained different results (compare dicussion section).

\subsection{The CREW model}

By inserting the derived closure relations into the REW scale mass balance equations, Eq. (1) to (11), we obtained the basic model equations of the CREW model (Cooperative Community Catchment model based on the Representative Elementary Watershed approach, Lee et al., $2006 b^{1}$ ). The 13 balance equations for mass and momentum are reduced to 9 with the aid of a series of assumptions used in Reggiani et al. (2000), which help exclude the momentum balance in the horizontal direction in the two subsurface zones. The resulting equations, including the new closure relations, can now be summarized as follows:

Unsaturated zone mass balance equation

$$
\begin{gathered}
\underbrace{\frac{d}{d t}\left(y^{u} \varepsilon^{u} \omega^{u} s^{u}\right)}_{\text {storage }}=\underbrace{\min \left[i \omega^{u}, \omega^{u} \overline{K_{s}}\left(1+\alpha^{u c} \frac{|\Psi|\left(1-s^{u}\right) \varepsilon^{u}}{s^{u} y^{u}}\right)\right]}_{\text {infiltration }}+\underbrace{\alpha^{u s} \omega^{u} v_{z}^{u}}_{\text {recharge or capillary rise }} \\
-\underbrace{\min \left[\omega^{u}\left(e_{p}+M k_{v} \overline{e_{p}}\right), \alpha_{w g}^{u} \frac{\omega^{u} \overline{K_{s}}}{\left(1-s^{u}\right) y^{u}} \frac{\left(s^{u}\right)^{2+d} \varepsilon^{u}\left|\Psi_{b}\right|}{m}\right]}_{\text {evapotranspiration }}
\end{gathered}
$$

Saturated zone mass balance equation

$$
\underbrace{\frac{d}{d t}\left(\varepsilon^{s} y^{s} \omega^{s}\right)}_{\text {storage }}=-\underbrace{\alpha^{u s} \omega^{u} v_{z}^{u}}_{\text {recharge or capillary rise }}-\underbrace{\omega^{o} \alpha_{1}^{o s}{\overline{K_{s}}}_{2}^{\alpha_{2}^{o s}}\left[\frac{y^{u} s^{u} \omega^{u}+y^{s}}{Z|\Psi|}\right]^{\alpha_{3}^{o s}}}_{\text {seepage }}-\underbrace{q_{s}}_{\text {sat. zone-river exchange }}
$$

Concentrated overland flow zone mass balance equation

$$
\underbrace{\frac{d}{d t}\left(y^{c} \omega^{c}\right)}_{\text {storage }}=\underbrace{\omega^{c} J}_{\text {rainfall or evaporation }}-\underbrace{\min \left[i \omega^{u}, \omega^{u} \overline{K_{s}}\left(1+\alpha^{u c} \frac{|\Psi|\left(1-s^{u}\right) \varepsilon^{u}}{s^{u} y^{u}}\right)\right]}_{\text {infiltration }}-\underbrace{\alpha^{o c} \xi^{r} y^{c} v^{c}}_{\text {flow to saturated overland flow zone }}
$$

Saturated overland flow zone mass balance equation

$$
\underbrace{\frac{d}{d t}\left(y^{o} \omega^{o}\right)}_{\text {storage }}=\underbrace{\omega^{o} \alpha_{1}^{o s} \bar{K}_{s}^{\alpha_{2}^{o s}}\left[\frac{y^{u} s^{u} \omega^{u}+y^{s}}{Z|\Psi|}\right]^{\alpha_{3}^{o s}}}_{\text {seepage }}+\underbrace{\alpha^{o c} \xi^{r} y^{c} v^{c}}_{\text {inflow from conc. overl. flow }}+\underbrace{\omega^{o} J}_{\text {rainfall or evaporation }}-\underbrace{\alpha^{r o} \xi^{r} y^{o} v^{o}}_{\text {lateral channel inflow }}
$$

Channel zone mass balance equation

$$
\underbrace{\frac{d}{d t}\left(m^{r} \xi^{r}\right)}_{\text {storage }}=\underbrace{\alpha^{r o} \xi^{r} y^{o} v^{o}}_{\text {lateral channel inflow }}+\underbrace{q_{s}}_{\text {sat. zone-river exchange }}+\underbrace{\sum_{l} \frac{m_{l}^{r} v_{l}^{r}}{\Sigma}}_{\text {inflow }}-\underbrace{\frac{m^{r} v^{r}}{\Sigma}}_{\text {outflow }}+\underbrace{\xi^{r} w^{r} J}_{\text {rainfall, evaporation on free surface }}
$$

\footnotetext{
${ }^{1}$ Lee, H., Sivapalan, M., and Zehe, E.: Cooperative Community Catchment model based on the Representative Elementary Watershed approach: numerical model development, benchmark tests and an application, Water Resour. Res., submitted, $2006 \mathrm{~b}$.
} 
$v_{z}^{u}=\frac{\bar{K}}{y^{u}} s^{u}\left[|\Psi|-\frac{1}{2} y^{u}\right]$

$v^{c}=\frac{1}{n_{m}^{c}}\left[y^{c}\right]^{\frac{2}{3}}\left[\sin \left(\gamma^{c}\right)\right]^{\frac{1}{2}}$

$v^{o}=\frac{1}{n_{m}^{o}}\left[y^{o}\right]^{\frac{2}{3}}\left[\sin \left(\gamma^{o}\right)\right]^{\frac{1}{2}}$

$v^{r}=\frac{1}{n_{m}^{r}} \sqrt{\frac{\left[\overline{R^{r}}\right]^{\frac{1}{3}}}{P^{r} l^{r}}\left[m^{r} l^{r} \sin \left(\gamma^{r}\right) \pm \sum_{l}\left\{\frac{1}{4} y^{r}\left(m^{r}+m^{l}\right) \cos \delta_{l}\right\}-\frac{1}{2} y^{r} m^{r}\right]}$

Please note that in doing so, the momentum balance Eq. (8), with respect to the unsaturated zone velocity, $v_{z}^{u}$, has been rewritten as Eq. (34), following the procedure adopted by Reggiani et al. (2000). The momentum balance equations for the c-, o-, and r-zones, i.e., Eqs. (9), (10) and (11), respectively, have been simplified as Eqs. (35), (36) and (37), by adopting the kinematic wave approximation, i.e., by ignoring the inertial term, and by adopting the relationship between Darcy-Weisbach friction factor and Manning coefficient, $\xi_{f}^{i}=8 g\left(n_{m}^{i}\right)^{2}\left(\overline{R^{i}}\right)^{\frac{1}{3}}, i=c, o, r$, for the second order friction term, $U^{i}, i=c, o, r$. The other procedure, which is necessary to convert the momentum balance equation for the channel reach, Eq. (11), into Eq. (37), is presented in Reggiani et al. (2001). Thus, Eqs. (35) and (36) are the REWscale Manning's equation for the movement over c- and ozones, respectively, while Eq. (37) is the REW-scale diffusive wave equation for channel flow.

Within the CREW model the balance equations for mass and momentum are solved by the adaptive Runge-Kutta integration method (Press et al., 1992). The current version of CREW model includes a total of 23 parameters: 8 parameters from closure relations $\left(\alpha^{u s}, \alpha_{1}^{o s}, \alpha_{2}^{o s}, \alpha_{3}^{o s}, \alpha^{u c}\right.$, $\left.\alpha_{w g}^{u}, \alpha^{o c}, \alpha^{r o}\right), 7$ parameters from constitutive relations $\left(\beta_{1}^{\omega^{o}}\right.$, $\left.\beta_{2}^{\omega^{o}}, \beta_{3}^{\omega^{o}}, \beta_{1}^{|\Psi|}, \beta_{2}^{|\Psi|}, \overline{K_{s}}, \beta_{2}^{\bar{K}}\right), 3$ Manning roughness coefficients $\left(n_{m}^{c}, n_{m}^{o}, n_{m}^{r}\right)$, porosities of the unsaturated and saturated zone $\left(\varepsilon^{u}, \varepsilon^{s}\right)$, canopy density $(M)$, the saturated hydraulic conductivity of the saturated zone $\left(\overline{K_{s}^{s}}\right)$, and the ratio of potential rates of transpiration and soil surface evaporation $\left(k_{v}\right)$. All parameters are allowed to be variable across REWs so that the effects of different soil textures, vegetation and geometries across REWs could be taken into account. To run the CREW, the required input information is climate data (rainfall, potential evaporation, and streamflow), and geometric information (length of channel reach at each REW, area of each REW, topographic slopes of the c-, o-, and rzones, total soil depth, elevation of channel bed from the datum, and the local angle between channel reachs of two neighbouring REWs). Information regarding to soil textures and vegetation can be imported into the modeling procedure by the relevant parameters. Currently, topographic slopes of the c-, o-, and r-zones are calculated based on the following equation, after Reggiani et al. (1999).

$\gamma^{i}=\cos ^{-1}\left(\frac{\Sigma^{i}}{S^{i}}\right), \quad i=c, o, r$

where $\Sigma^{i}$ is projected area of the $i$-zone onto the horizontal plane and $S^{i}$ is the surface area of $i$-zone. $S^{i}$ was identified by following the slope in the direction of steepest descent for each grid cell within the digital elevation model and the same $S^{i}$ value was used for the c-, and o-zones. However, it should be pointed out that topographic slopes of the c-, o-, and r-zones in Eq. (35) to (37) are effective values defined at the REW scale and they are introduced to account for the balance of forces at the REW scale. Therefore, topographic slopes $\gamma^{i}$ should be calculated in a way that they assure balance of momentum in the averaging process, and, at the same time, reflect local geometries of the study area. This may give rise to the problem of parameter estimation by considering $\gamma^{i}$ as one of parameters controlling especially the flow routing process.

\subsection{Numerical test of the CREW model}

Two numerical experiments were designed to see how the derived closure relations respond to combinations of climate, soil, vegetation and topography, within the REW modelling framework. The values of the parameters, input data and initial conditions used in the numerical experiments are summarized in Table 3. Sensitivity analyses of infiltration and infiltration excess surface runoff generation processess were the 
first test, because infiltration excess surface runoff is known as the dominant runoff generation mechanism in Weiherbach catchment (Zehe and Blöschl, 2004). The second test was designed to explore streamflow at the outlet as an integrated catchment response combining many interacting processes. Sensitivity analyses were conducted by changing one variable at a time, in each case. Various numerical tests to assess the hydrological functioning produced by the coupled balance equations of the REW approach can be found at Lee et al. (2005a).

3.5.1 Infiltration and infiltration excess surface runoff generation

This test is designed to see the effect of antecedent moisture content (AMC), rainfall intensity, and different soil types on the infiltration rates, and infiltration excess surface runoff resulting from different rainfall intensities. The effect of AMC on infiltration is modelled by assigning different initial soil moisture contents to the unsaturated zone, while all other inputs are parameters are held fixed. To see the effect of rainfall intensity on infiltration rate and infiltration excess surface runoff, rainfall intensities are varied, while other information such as soil type, AMC etc. are held fixed. Likewise, different soil types are used to see their effects on the infiltration excess runoff process, while other variables held fixed.

For sensitivity analyses of the closure relationship for infiltration, the fixed values chosen are $D I=0.5, t_{r}=2$ [day], $t_{b}=8$ [day], $M=0, k_{v}=1, n_{m}^{c}=0.07\left[\mathrm{~m}^{-1 / 3} \mathrm{~s}\right], n_{m}^{o}=0.035\left[\mathrm{~m}^{-1 / 3} \mathrm{~s}\right]$, $n_{m}^{r}=0.03\left[\mathrm{~m}^{-1 / 3} \mathrm{~s}\right], q_{s}=0.00012[\mathrm{~mm} / \mathrm{hr}], Z=8[\mathrm{~m}], z^{r}=21$ $[\mathrm{m}], z^{s}=20[\mathrm{~m}], \beta_{1}^{\omega^{o}}=0.3, \quad \beta_{2}^{\omega^{o}}=0.3, \quad \beta_{3}^{\omega^{o}}=0.4, \quad \alpha^{u c}=0.1$, $\alpha_{w g}^{u}=5, \alpha^{u s}=1, \alpha^{o c}=1.5, \alpha^{r o}=2.5, \alpha_{1}^{o s}=10, \alpha_{2}^{o s}=6.2, \alpha_{3}^{o s}=2.7$ where $D I$ is the ratio of total annual potential evaporation to total annual precipitation, called the climatic dryness index. In order to fully test the infiltration model, larger unsaturated zone depths are used than in the subsequent test.

Figure 7a shows the effect of AMC of the unsaturated zone on the infiltration rates, for a silty loam, with constant rainfall intensity of $20[\mathrm{~mm} / \mathrm{hr}]$. The results show that as AMC increases, the infiltration rate decreases, the infiltration capacity is higher than rainfall intensity for the smallest AMC used, and that the infiltration rate decreases exponentially after the surface is ponded, which is a well known infiltration behaviour (cf., Sivapalan and Wood, 1986). Figure 7b shows the effect of rainfall intensity, again for a silty loam, when the AMC is zero. The results show that the bigger the rainfall intensity is, the less the time to ponding is, and that regardless of rainfall intensity, the infiltration capacity approaches the same asymptotic value at large time. At lower precipitation intensities, i.e., 1,5 and $10[\mathrm{~mm} / \mathrm{hr}]$, all precipitation is infiltrated since the infiltration capacity is greater than the rainfall intensity. Figure $7 \mathrm{c}$ shows the effect of different soil types, silty loam (solid line) and sand (circle), under different rainfall intensities, and zero AMC. Infiltration rate is very high for sandy soils, which is the result of high infiltration capac- ity. This is confirmed by perusing the infiltration capacity Eq. (13), in the light of the soil properties presented in Table 3. The hydraulic conductivity of sand is much higher than that of silty loam, even though bubbling pressure head and porosity are low, which makes the infiltration capacity to be rather high. The infiltration rate for sand at large times decreases smoothly, not exponentially, when rainfall intensity is $40[\mathrm{~mm} / \mathrm{hr}]$. This is different from the results presented in Fig. $7 \mathrm{a}$, and is due to the decrease of the unsaturated area fraction by water table rise, and not due to the occurrence of surface ponding.

Figure $7 \mathrm{~d}$ shows Hortonian overland flow corresponding to Fig. 7b. Hortonian overland flow accompanies the infiltration process across the concentrated overland flow zone. This is reproduced in Figs. 7b and d. At the end of the storm, i.e., $t>t_{r}$ Hortonian flow ceases abruptly due to cessation of rainfall. Note here that storm period $t_{r}$ is defined as the period over which an event lasts without ceasing in the middle; in this study, we used constant values for the storm period along with constant rainfall intensities. On the whole, the water flow dynamics within the concentrated overland flow zone is qualitatively well captured by the adopted closure relations for infiltration capacity and concentrated overland flow, even though the exact magnitude may not be accurate since there has not been any calibration involved, and the question of estimating appropriate parameters remains to be accomplished.

\subsubsection{Integrated catchment response measured at the outlet}

Figure 8 presents the breakdown of various processes occurring over the catchment in response to a constant intensity event. These include seepage outflow, saturated overland flow and channel flow. The rainfall input is $10[\mathrm{~mm} / \mathrm{hr}]$, and the soil type used was sand. The fixed values are $D I=0.5, t_{r}=2$ [day], $t_{b}=8$ [day], $M=1, k_{v}=1$, $n_{m}^{c}=0.07\left[\mathrm{~m}^{-1 / 3} \mathrm{~s}\right], n_{m}^{o}=0.035\left[\mathrm{~m}^{-1 / 3} \mathrm{~s}\right], n_{m}^{r}=0.03\left[\mathrm{~m}^{-1 / 3} \mathrm{~s}\right]$, $q_{s}=0.00012[\mathrm{~mm} / \mathrm{hr}], Z=8[\mathrm{~m}], z^{r}=25[\mathrm{~m}], z^{s}=20[\mathrm{~m}]$, $\beta_{1}^{\omega^{o}}=0.3, \quad \beta_{2}^{\omega^{o}}=0.3, \quad \beta_{3}^{\omega^{o}}=0.4, \quad \alpha^{u c}=1, \quad \alpha_{w g}^{u}=100, \quad \alpha^{u s}=1$, $\alpha^{o c}=1.5, \alpha^{r o}=2.5, \alpha_{1}^{o S}=2000, \alpha_{2}^{o s}=5.2, \alpha_{3}^{o s}=2.7$. We see in Fig. 8 that the total saturated overland flow is a combined response of both seepage flow and rainfall falling on saturated areas. There was no Hortonian overland flow from the concentrated overland flow zone in this case, so it can be inferred that the decline of infiltration flux displayed is not caused due to the reduced soil infiltration capacity or surface ponding, but rather caused by the increased saturated area fraction. This is confirmed by the surface runoff caused by rainfall falling on saturated areas. In Fig. 8, the discharge hydrograph at the catchment outlet is almost the same as saturated overland flow, and does not show any effect of channel storage. This is partly due to the size of catchment used, and the nature of closure relations used for channel flow. The generalization of these closure relations to reflect dynamic 


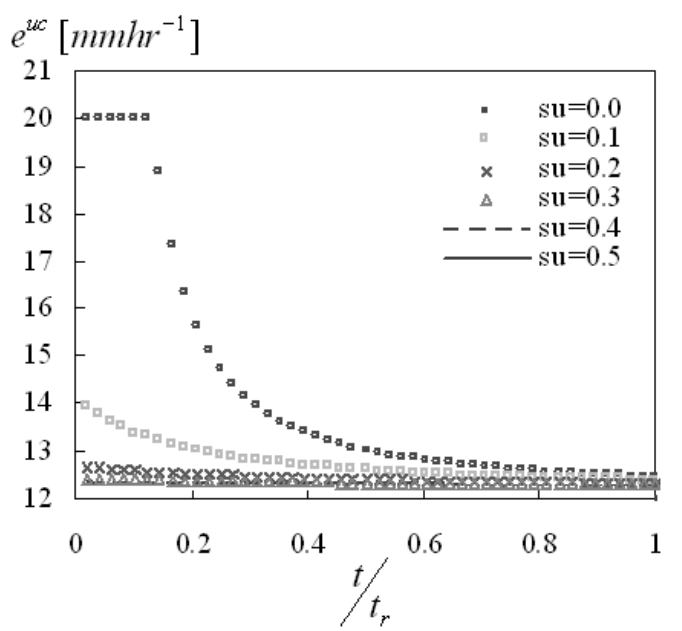

(a)

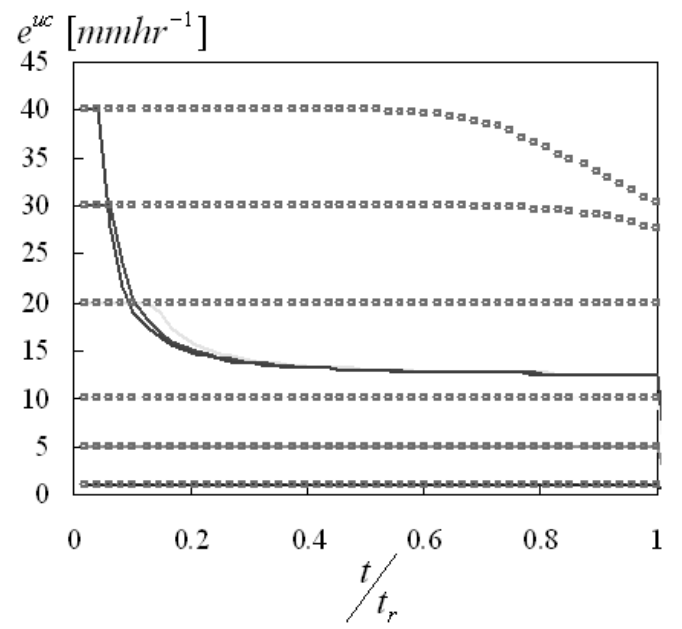

(c)

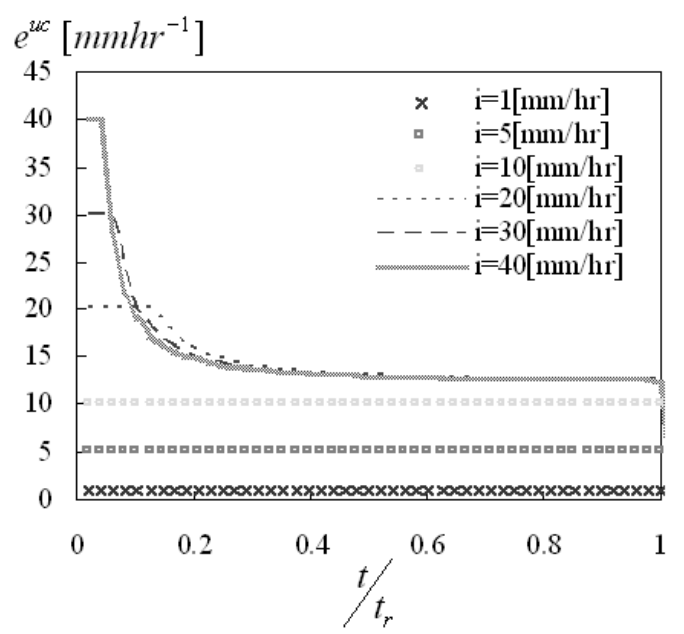

(b)

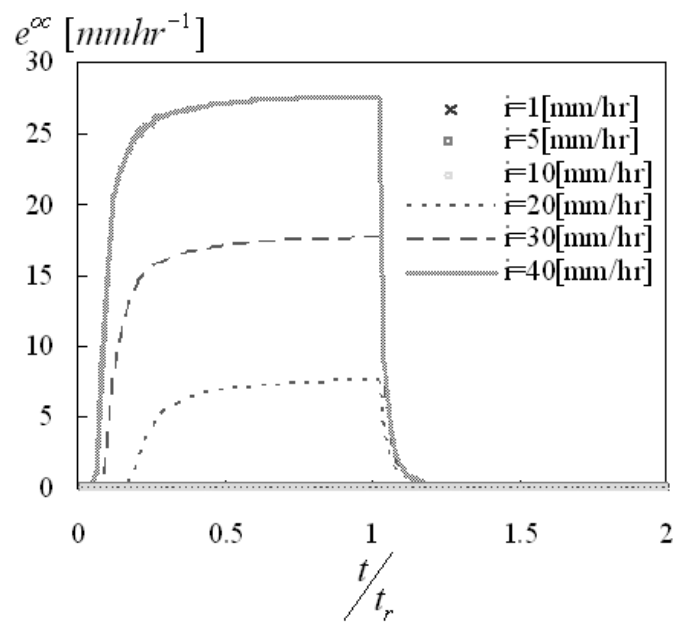

(d)

Fig. 7. Sensitivity analysis on closure relation for infiltration and concentrated overland flow (a) the effect of antecedent moisture content on the closure relation for infiltration process: silty loam (b) climate effect on the closure relation for infiltration process: silty loam (c) the effect of different soil on the closure relation for infiltration rate: solid line for silty loam, circle for sand (d) climate effect on the closure relation for concentrated overland flow: silty loam.

effects, including diffusion and inertial effects is the subject of future work.

\section{Application of the CREW model to the Weiherbach cachment}

\subsection{Design of simulation tests and set up of the CREW} model

Finally we want to shed light on the question whether the proposed closure relations and constitutive relations allow reasonable predictions when the CREW model is setup for the Weiherbach catchment. To this end the time series of average soil moisture predicted by CREW is compared against the time series of volume integrated soil moisture simu- lated by the fully distributed model, CATFLOW. The model structure employed is based on 169 typical hillslope and a drainage network and yielded, as already stated, good predictions of discharge and ET, and soil moisture measured at 61 TDR stations. The volume integrated soil moisture from this model structure is hydrologically consistent with this observation and also consistent with the typical patterns observed in this catchment. It can therefore be deemed as the best guess of how the true average soil moisture in the Weiherbach catchment evolved under the boundary conditions within the simulation period and an interesting benchmark for CREW. In addition we employed discharge data aggregated to the daily scale as well as the observed soil moisture at the 61 TDR stations as measures to judge the performance of CREW. 
Table 3. Values of parameters, input data and initial conditions used in the sensitivity analysis.

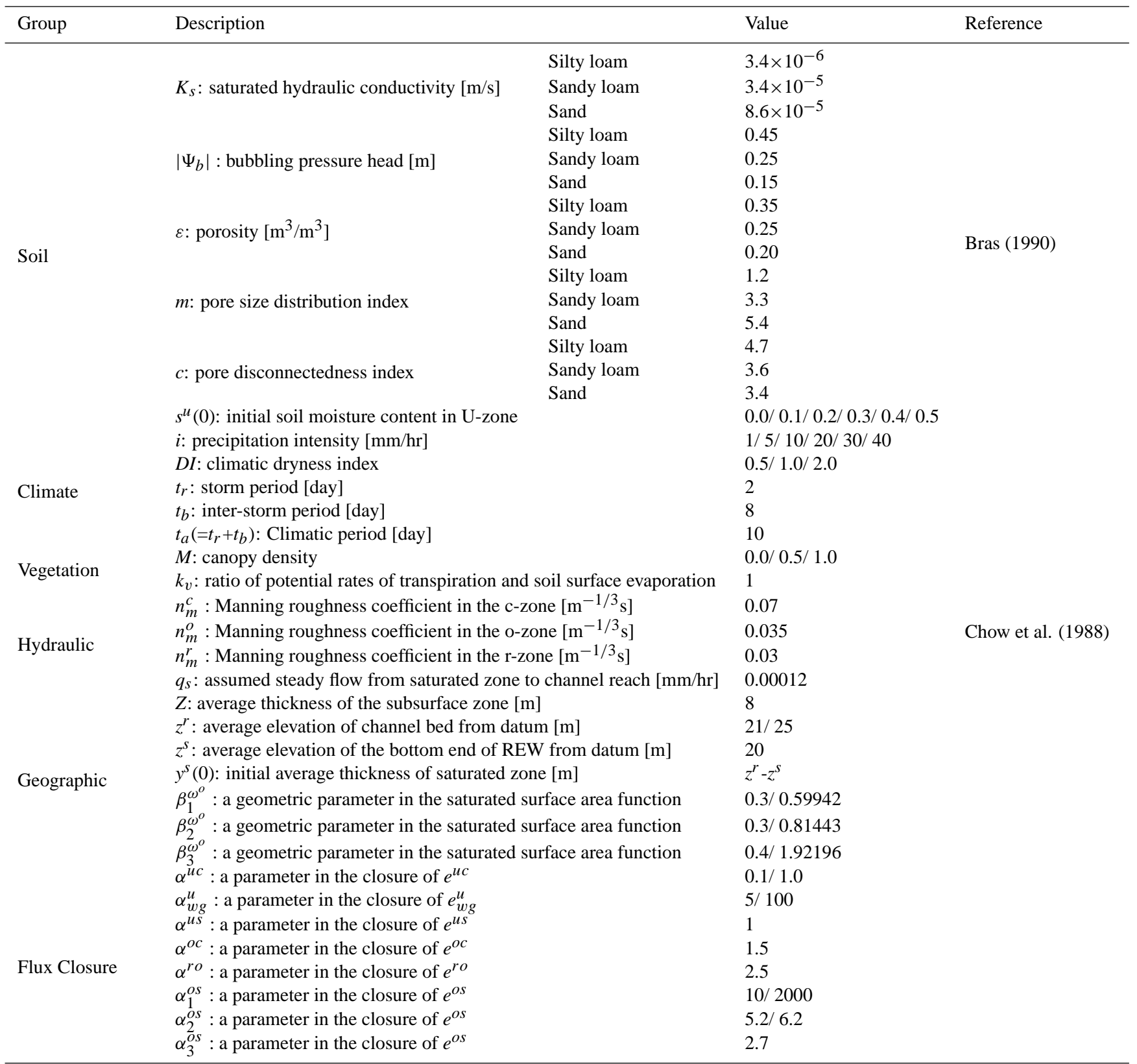

Unlike CATFLOW, which uses an advanced SVAT approach based on the Penman-Monteith equation, CREW needs potential evaporation as input for determining actual evaporation; for this reason, hourly potential evaporation data were generated for the Weiherbach catchment based on the results of Zehe et al. (2001) to be used as input for the CREW model. The initial saturation of the soil in the u-zone was set at 0.5 for both CATFLOW and CREW and the simulations were carried out for the periond of 21/04/1994 to 20/04/1995 with identical time series of precipitation data, except that the data for CREW were aggregated from $6 \mathrm{~min}$ to $1 \mathrm{~h}$ resolution. The boundary conditions were chosen in both models to be the atmosphere at the top and such that seepage flux was not allowed. Slopes of the c-, o-, and r-zones are calculated using Eq. (38) with the use of digital elevation model (DEM) of the catchment. Unsaturated zone depth used was 2 metres for both CREW and CATFLOW. Canopy density $(M)$ was assumed to be equal to 1.0 (unity) for the CREW run, i.e., fully vegetated during the year. Within CREW we used parameter values directly, with- 


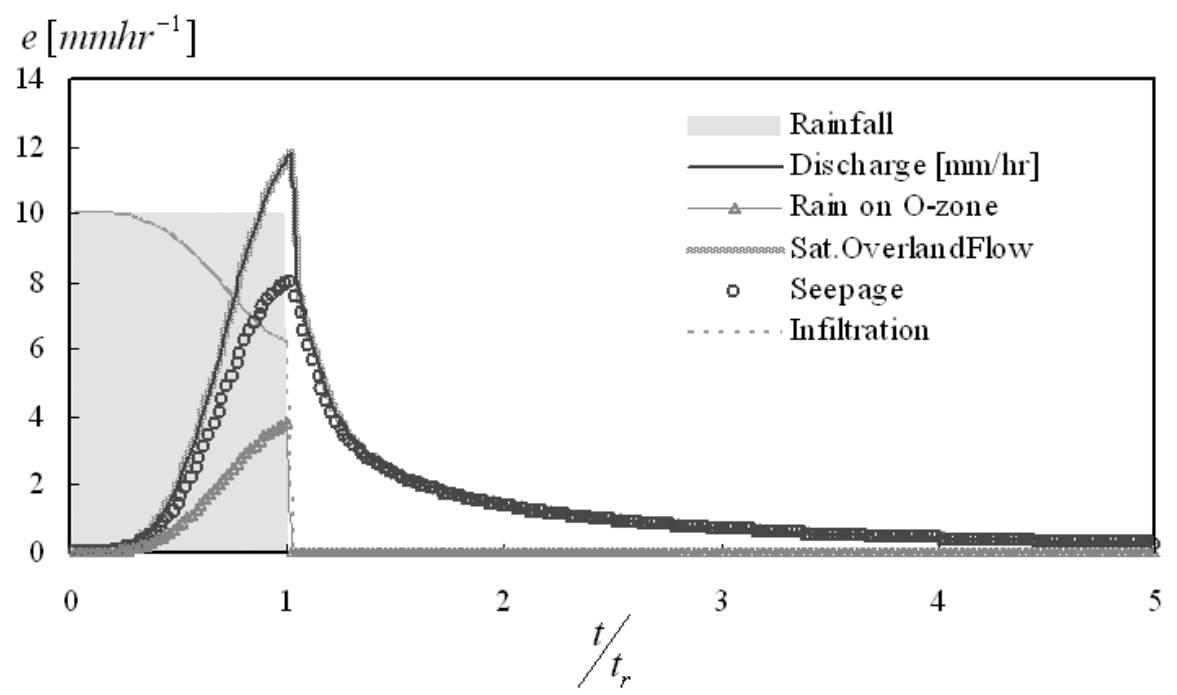

Fig. 8. Saturated overland flow and discharge at the channel outlet as the integrated response of all processes happening in the catchment.

out adjustment, obtained from Weiherbach catchment during the upscaling procedure used to develop closure relations and constitutive relations $\left(\alpha^{o c}=1.0, \alpha^{r o}=1.0, \beta_{1}^{\omega^{o}}=0.71\right.$, $\left.\beta_{2}^{\omega^{o}}=1.79, \beta_{3}^{\omega^{o}}=0.92\right)$. The parameter values estimated here and for the constitutive relations are shown in Table 4.

\subsection{Simulation results}

In the absence of calibration the CREW model did not yield good results for soil moisture or for discharge. The REW scale saturated hydraulic conductivity $\overline{K_{s}}$, the related parmeters of the REW "textural" hydraulic functions and $\alpha_{w g}^{u}$ turned out to be inappropriate. Hence we allowed for manual calibration of the parameters $\beta_{1}^{|\Psi|}, \beta_{2}^{|\Psi|}, \beta_{2}^{\bar{K}}$ and $\alpha_{w g}^{u}$ in the sense that the model should match the largest event observed and at the same time the rescession. The following parameter $\beta_{1}^{|\Psi|}=0.21 \mathrm{~m}, \beta_{2}^{|\Psi|}=0.25$ (water retention curve), $\overline{K_{s}}=8.0 \times 10^{-6}, \beta_{2}^{\bar{K}}=4.51$ (hydraulic conductivity curve) and $\alpha_{w g}^{u}=15$ yielded a Nash-Sutcliffe efficiency that was larger than 0.8 which is denoted as "simM", the case without calibration is denoted as "simMC".

Figure 9a shows that the temporal $s^{u}$ time series simulated by CREW and that the volume integrated saturation values simulated with CATFLOW are in very good agreement. The CREW simulation falls within the range of observed soil moisture values, which were obtained with $60 \mathrm{~cm}$ two rod TDR sensors during the entire period, as shown in Fig. 9b. The observed soil moisture is largely scattered which reflects the high spatial variabilities at the point scale. Maximum and minimum observed saturation values over a given time step could be used to define the limits of simulated $s^{u}$ over the same time step. The fact that the CREW simulation falls within the range of observed soil moisture values alone would not be too exciting. However, the additional good match between the CREW results and the integrated soil saturation values from CATFLOW suggest that CREW produces realistic unsaturated zone saturation values at this scale.

Figure 10 compares observed streamflows $\left(Q_{\text {obs }}\right)$, with those simulated by CREW using with $\left(Q_{\text {simM }}\right)$ and without additional calibration $\left(Q_{\text {simMC }}\right)$. We chose two flood events to highlight the differences between the two models structures, as shown in Figs. 10b and c. Peak flow for event 1 shown in Fig. 10b is quite well captured by both CREW simulations. During small rainfall events, $Q_{\text {simMC }}$ did not capture peak flows well, as shown in Fig. 10c for event 2.

Runoff contributions by different mechanisms for events 1 and 2 by both simulations are summarized in Figs. 10d and e. Runoff during event 1 was mostly produced by infiltration excess overland flow (92 and 94\% of streamflow in case of $Q_{\text {simM }}$, and $Q_{\text {simMC }}$ respectively), which is consistent with previous results on this catchment (Zehe et al., 2005b). Peak flow produced by $Q_{\text {simM }}$ during event 2 was mainly generated by saturation excess overland flow, and contribution of infiltration excess overland flow to peak streamflow was not significant in this case. In the case of $Q_{\text {simMC }}$ during event 2 there was very small saturation excess overland flow simulated $(0.2 \%$ of streamflow). This can be explained by a smaller saturated surface area resulting from the three parameters related to Weiherbach catchment geometries $\left(\beta_{1}^{\omega^{o}}, \beta_{2}^{\omega^{o}}\right.$, and $\beta_{3}^{\omega^{\circ}}$ ) used to estimate saturated surface area as a function of average vertical thickness of saturated zone. For both models streamflow was mainly fed by subsurface flow. In both model simulations during event 2 , no infiltration excess overland flow occurred due to the high infiltration capacity in comparison to the rainfall intensity. However, in this catchment, the main storm runoff generation mechanism has been previously assumed to be infiltration excess overland flow 


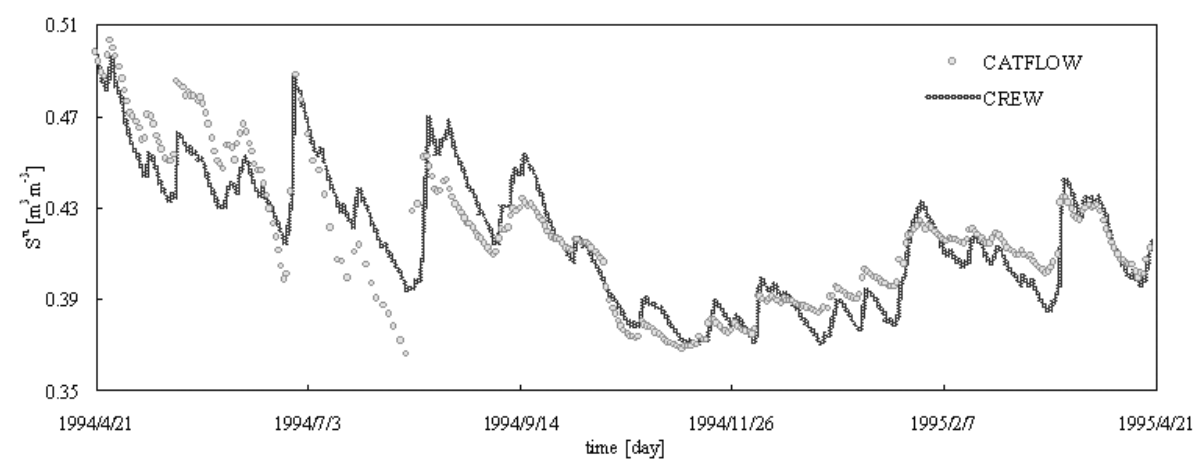

(a)

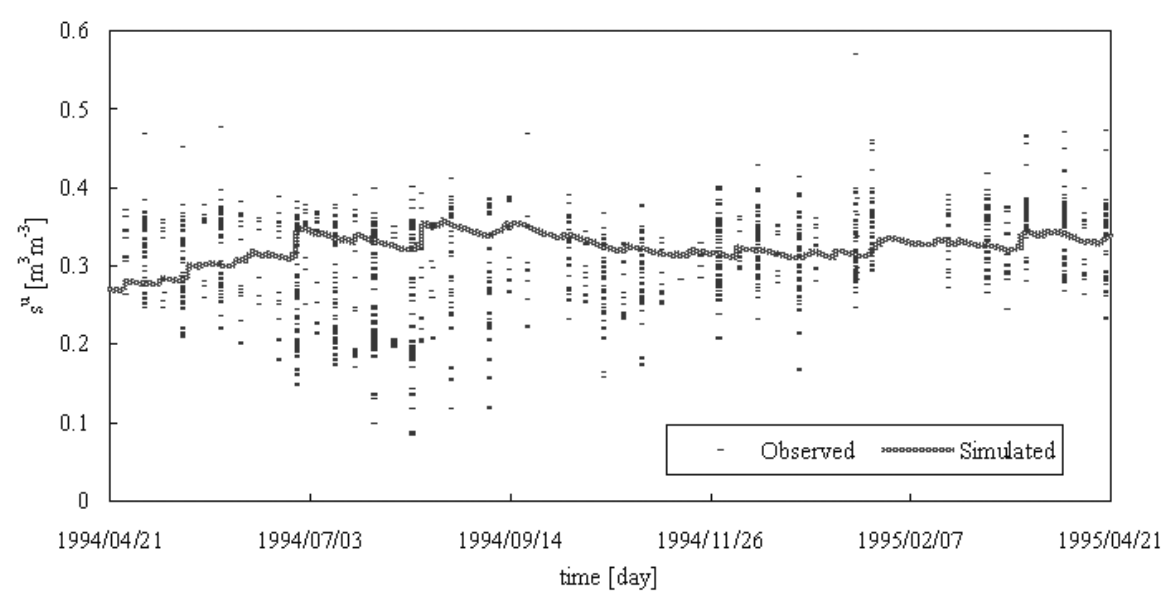

(b)

Fig. 9. (a) Comparison of catchment scale saturation degree in the unsaturated zone, $s^{u}$, simulated from CATFLOW and CREW, and (b) comparison of $60[\mathrm{~cm}]$ depth averaged measured surface soil moisture content with saturation degree in the unsaturated zone, $s^{u}$, simulated by CREW.

(Zehe et al., 2005b; Zehe and Blöschl, 2004). Our results indicate that it might be necessary to revisit the closure relations or that the conclusions of the latter authors were wrong for smaller events.

Figure 11 shows discharge time series for both events simulated by the CREW model using an hourly time step with parameters that have been calibrated on daily discharge values. Clearly, the parameters estimated at the daily scale produce timing problems when applied at the hourly time scale, with CREW responding too quickly to rainfall events $(1 \mathrm{~h})$, which we suspect could be due to an overestimation of average slopes by the simple geometric approach that we employed here. This requires further investigation, and is examined in detail in a subsequent study (Lee et al. $\left.2006 \mathrm{a}^{2}\right)$ involving the application of CREW to mesoscale catchments, where the timing is absolutely critical.

\section{Discussion and conclusions}

In the context of the REW approach of Reggiani et al. (1998, 1999), the development of physically reasonable closure relations for various mass exchange fluxes is a crucial step to ground REW approach to reality and to make models based on the REW approach applicable to real world catchments (Beven, 2002). Due to the lack of direct observation techniques at that scale, the best way to start with this problem is to start at a catchment that offers a wide range of data, that is well understood and where finer scale models have been employed to support this process. This is the case for the Weiherbach catchment, which provided the catalyst to push the development of CREW and the related closure relations so far, that we could come up with the first application of this model to a real catchment.

\footnotetext{
${ }^{2}$ Lee, H., Sivapalan, M., and Zehe, E.: Application of the distributed physically based model, CREW, to two mesoscale Australian catchments in contrasting climates, J. Hydrol., submitted, 2006a.
} 

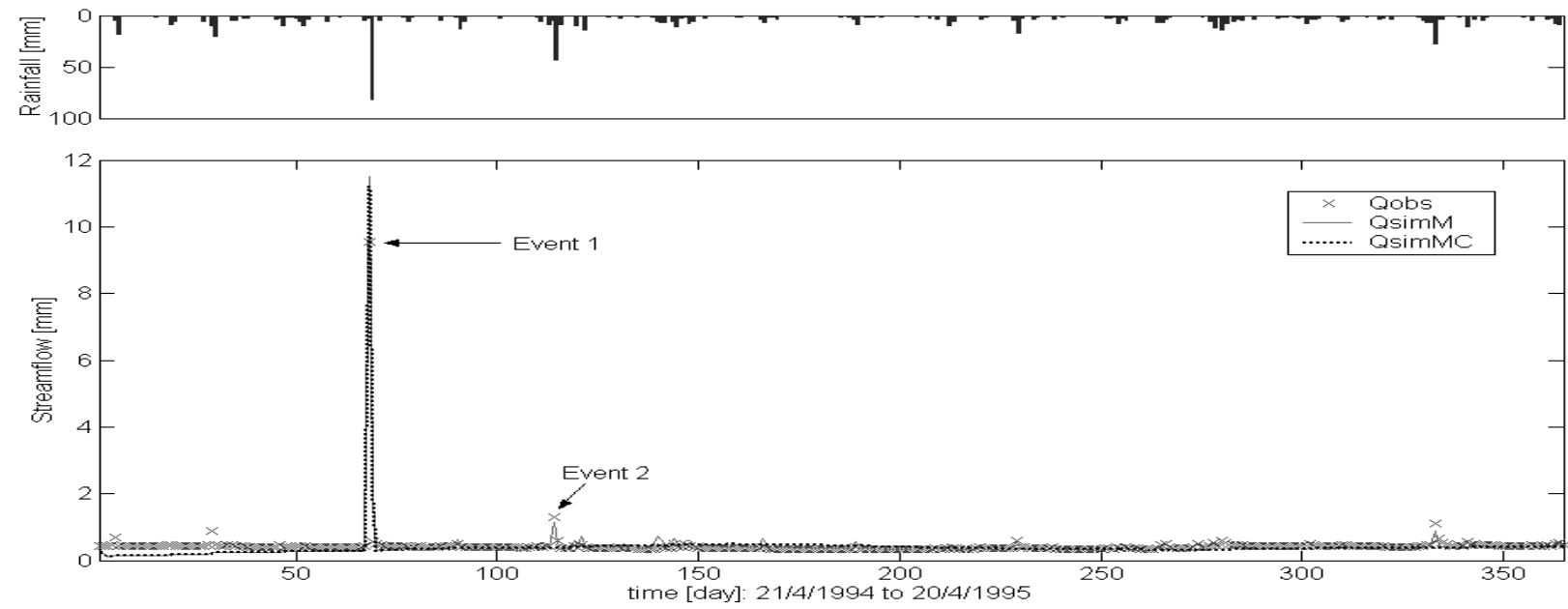

(a)
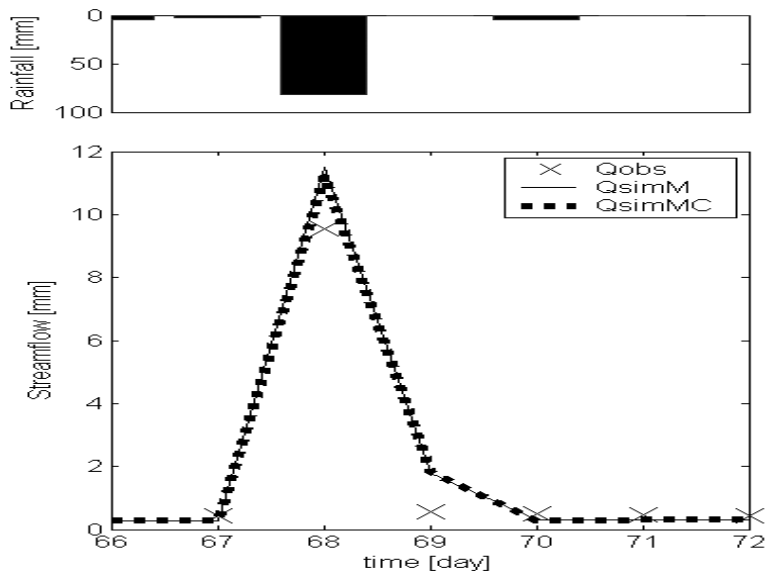

(b)

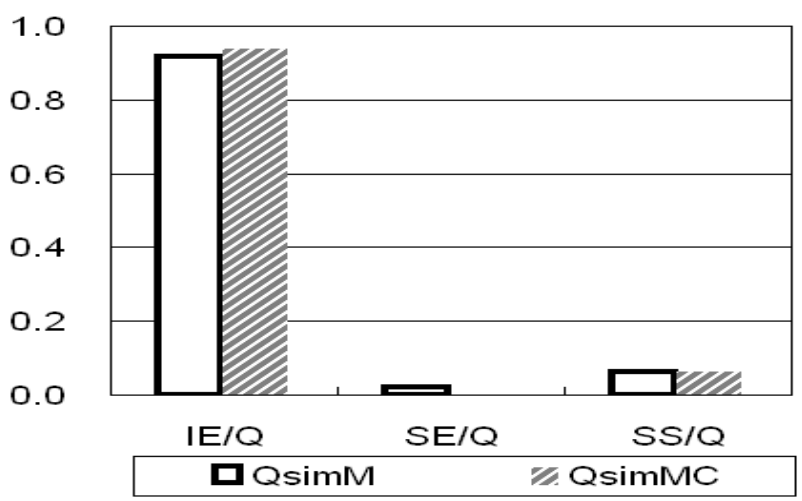

(d)
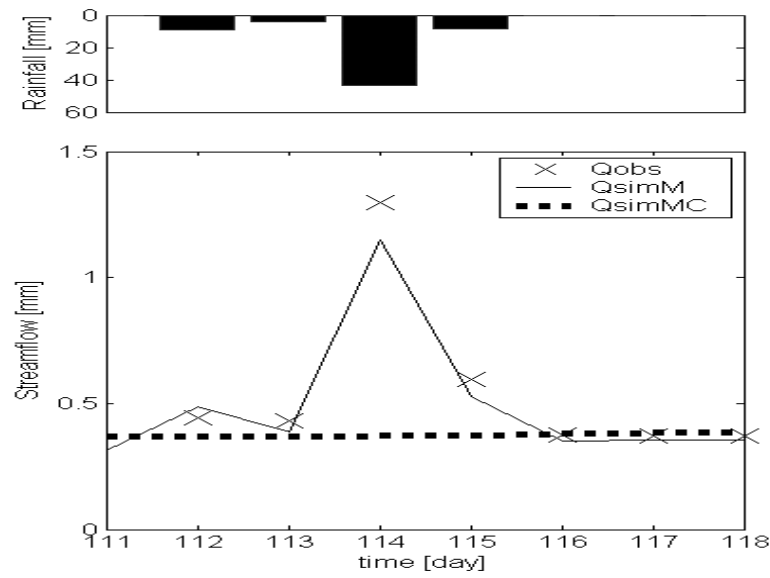

(c)

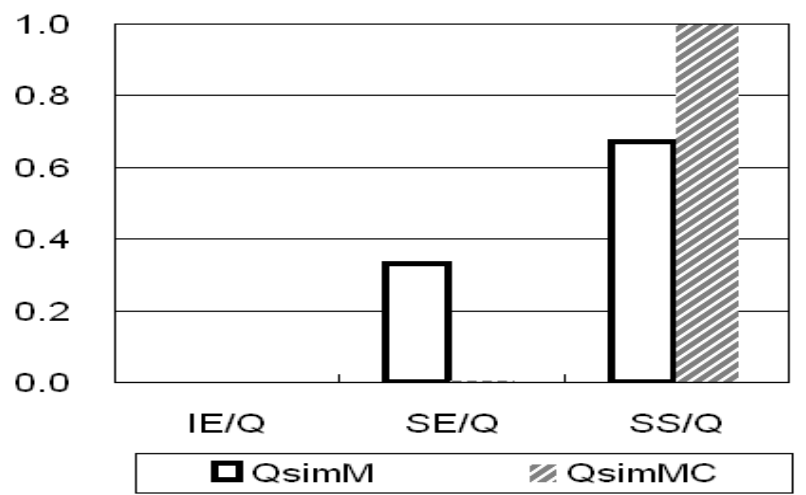

(e)

Fig. 10. Comparison of observed streamflows with those simulated by CREW at the daily time scale where $Q_{\text {obs }}, Q_{\text {simM }}$ and $Q_{\text {simMC }}$ denote the observed hydrograph, simulated hydrograph with manually calibrated parameter values, and simulated hydrograph with both closure parameters and manually calibrated ones, respectively: daily rainfall and streamflow time series from Weiherbach catchment (a) from 21/4/1994 to 20/4/1995, (b) for event 1, and (c) for event 2, and runoff contribution of different hydrologic processes for (d) event 1, and (e) event 2 where IE, SE, SS, and Q denote infiltration excess, saturation excess overland flow, subsurface flow, and streamflow respectively. 
Table 4. Parameter values for the Weiherbach catchment estimated (a) from the upscaling procedure, (b) by manual calibration of CREW, and (c) by manual calibration of CREW as well as the upscaling procedure.

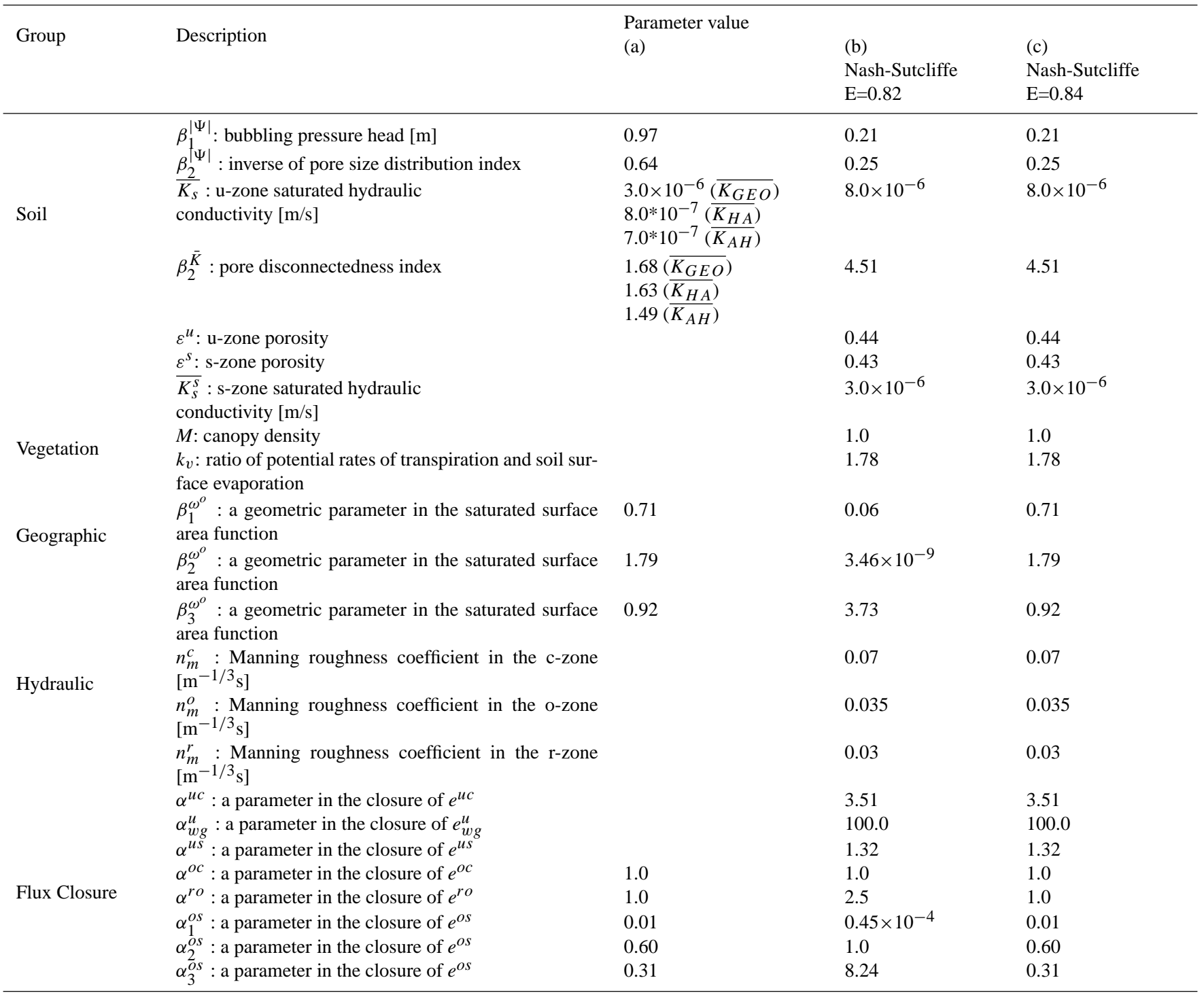

We derived closure relations for infiltration, exfiltration, groundwater recharge and capillary rise analytically using various assumptions regarding sub-grid heterogeneity. The closure relations for saturation excess overland flow and concentrated overland flow were derived by a combination of the analytical approach, but assisted by limited numerical models, i.e., by a hybrid approach. Finally, the closure relations for seepage flow, or subsurface stormflow, were derived by the application of the numerical simulation approach, with the use of the CATFLOW model. Following Zehe et al. (2005a) the full catchment scale model structure was also used to assess an REW-scale pressure-saturation relationship (i.e., water retention curve) and unsaturated hydraulic conductivity versus saturation relationship, in both cases the model was driven with observed atmospheric boundary conditions as explained in Sect. 3.3.2. Finally, a geometric relationship linking saturation area to saturated zone water depth was obtained based on TOPMODEL assumptions.

The first thing to state is that with the derivation of all of the closure relations and necessary geometric and constitutive relations, the REW scale balance equations are fully determinate and the CREW model has been born. A first sensitivity analyses based on literature data showed that the model produces realistic infiltration behaviour for different soils types, initial conditions and rainfall intensities. However, the real benchmark for CREW was the application to the Weiherbach catchment and the comparsion with observed soil moisture and discharge data as well as with the volume 

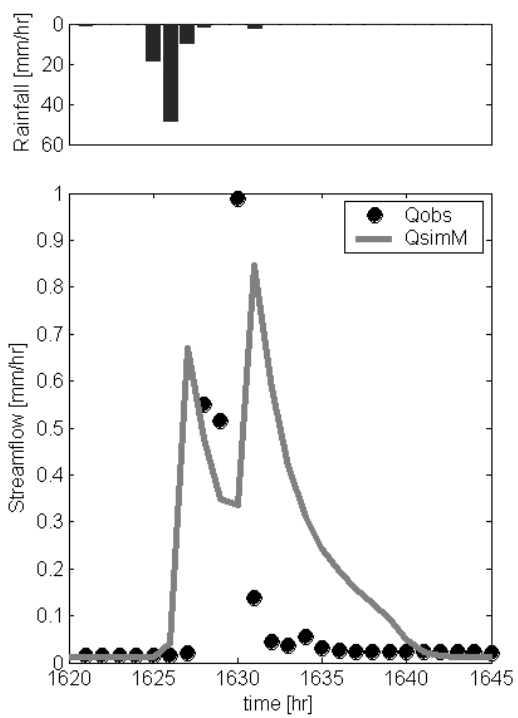

(a)
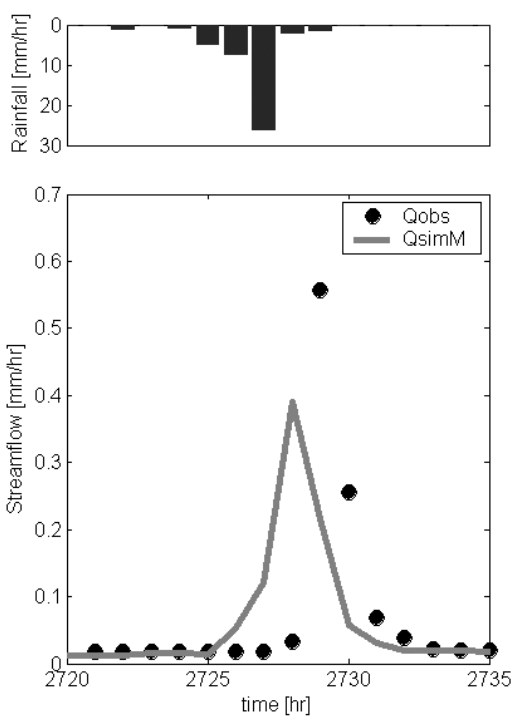

(b)

Fig. 11. Comparison of observed streamflows with those simulated by CREW at the hourly time scale where $Q_{\text {obs }}$ and $Q_{\text {simM }}$ denote the observed hydrograph and simulated hydrograph with manually calibrated parameter values respectively: hourly rainfall and streamflow time series from Weiherbach catchment (a) for event 1, and (b) for event 2.

integrated soil moisture simulated with CATFLOW. The latter can be deemed as best estimate of how the true average soil moisture evolved in the Weiherbach responding to the boundary conditions within the simulation period.

To match the observations/fine scale simulations we had to allow for additional calibration of parameters related to the REW scale water retention curve and unsaturated hydraulic conductivity curve. Hence, we must state that the numerical uspscaling we employed here for these properties did not fully work out. However, the REW scale saturated hydraulic conductivity value we obtained in the calibration is at $8 \times 10^{-6} \mathrm{~m} / \mathrm{s}$ similar to the REV scale values of the Weiherbach soils (Table 1). After the calibration the model yielded good results for daily discharges and at the same time for soil moisture. The CREW simulation of unsaturated zone saturation fell within the range of observed soil moisture and matched the integrated soil saturation values from CATFLOW very well. Therefore, we conclude that CREW is capable of reproducing internal state dynamics and discharge at least in the Weiherbach at the same time, something that is very rarely shown in hydrology.

While this is a major success, it would be stupid to claim that the closure problem is solved. This is just a start. Simulations at the hourly time scale showed that CREW responds too quickly, which provides the hint that the derivation of average slopes may be too simple. Secondly, CREW simulations suggest that a major contribution to discharge dur- ing the small event 2 is subsurface flow, which contradicts studies of Zehe et al. (2005b) in this catchment. However, also Zehe et al. (2005b) could be wrong with the assumption that Hortonian overland flow is always dominant in the Weiherbach catchment. Smaller events could stem just from the zone close to the brook where a shallow aquifer is present. Another serious shortcoming of the REW approach itself is for sure the zero dimensional approach for the unsaturated zone, as it does not allow the resolution into multiple layers, the root zone etc. Future research should focus on advancing the unsaturated zone by introducing several layers or even going back to a vertically distributed approach.

Concerning the derivation of constitutive relations it is interesting to note that the approach presented here did not work, but the approach suggested by Zehe et al. (2006, compare Sect. 3.3.2) a companion study yielded parameter values that were much closer to those obtained within the manual calibration process. Hence, it seems that further research on the dynamical upscaling is needed to clarify the limits of such an approach (in terms of heterogeneity of the system). The most important issue is to further advance observation techniques that might allow assessment of patterns and state variables over larger volumes, which could strongly support the development of closure relations. Geophysical techniques and tracers are certainly promising within this respect. Finally, there is also the problem of equifinality/predictive uncertainty (Beven and Freer, 2001), which has to be investi-

\footnotetext{
${ }^{3}$ Lee, H., Zehe, E., and Sivapalan, M.: Investigation of runoff prediction uncertainty and parameter sensitivity for the distributed model CREW using GLUE, Adv. Water Resour., submitted, 2006c.
} 
gated for models based on the REW approach. In this regard, recent more detailed studies by Lee et al. (2006c) $)^{3}$ addresses the issues of equifinality and runoff prediction uncertainty of CREW using GLUE.

Our final constructive comment is that we believe that models based on the REW approach are the most promising candidates for better hydrological modelling at the mesoscale. An REW together with its closure relations is functional unit. If there is something like a typical hydrological functioning of landscapes, then we believe this must be the case. It should be possible to derive typical REWs for a landscape. Models that are composed of such typical REWs could then be expected to have a lower structural uncertainty/ be more compatible with the landscape in the sense of Wagener et al. (2003) and yield much certain predictions.

\section{Appendix A}

\section{Derivation of a closure relation for bare soil evaporation and transpiration by root uptake $\left(e_{w g}^{u}\right)$}

One dimensional concentration dependent diffusion equation describing soil moisture movement in the unsaturated zone (Philip, 1960) has the following form, when it includes a soil moisture extraction term by root uptake as a sink (Eagleson, 1978a):

$$
\frac{\partial \theta}{\partial t}=\frac{\partial}{\partial t}\left[D(\theta) \frac{\partial \theta}{\partial z}\right]-\frac{\partial K(\theta)}{\partial z}-g_{r}(z, \theta)
$$

where $\theta$ is the effective volumetric moisture content, $t$ is time, $K(\theta)$ is the effective hydraulic conductivity, $D(\theta)$ is the diffusivity, $g_{r}(z, \theta)$ root extraction function, and $z$ is vertical coordinate. In analogy with the infiltration capacity equation of Philip (1960), Eagleson (1978b) derived an exfiltration capacity equation as:

$f_{e}^{*} \approx \frac{1}{2} S_{e} t^{-\frac{1}{2}}-M e_{v}$

where $f_{e}^{*}$ is exfiltration capacity for bare soil evaporation, $M$ is canopy density, and $e_{v}$ is transpiration rate. In (A2), $S_{e}$ is an exfiltration sorptivity defined by:

$S_{e}=2\left(\theta_{0}-\theta_{1}\right)\left[\frac{D_{e}}{\pi}\right]^{\frac{1}{2}}$

where $\theta_{0}$ is initial effective volumetric moisture content, $\theta_{1}$ is effective volumetric moisture content at the soil surface, and $D_{e}$ is a desorption diffusivity defined by:

$D_{e}=\frac{K_{s}\left|\Psi_{b}\right| s_{0}^{d} \phi_{e}}{m \varepsilon}$

where $K_{s}$ is the saturated hydraulic conductivity, $\left|\Psi_{b}\right|$ is bubbling pressure head, $s_{0}$ is initial degree of saturation at the soils surface, where this value is fixed by 'zeroth-order' approximation at a space-time average soil moisture (Eagleson, 1978a), $d$ is a diffusivity index, $m$ is the pore size distribution index, $\varepsilon$ is soil porosity, and $\phi_{e}$ is a dimensionless exfiltration diffusivity defined as:

$\phi_{e}=1.85 s_{0}^{-1.85-d} \int_{0}^{s_{0}} s^{d}\left(s_{0}-s\right)^{0.85} d s$

Now let us define combined evapotranspiration capacity, $f_{E T}^{*}$, by both bare soil evaporation and transpiration by root uptake, as follows:

$f_{E T}^{*}=f_{e}^{*}+M e_{v}$

If Eq. (A4) is substituted into Eq. (A3), with the condition $\theta_{1}$ is equal to zero for exfiltration, then we have:

$S_{e}=S_{e r} K_{s}^{\frac{1}{2}}$

$S_{e r}=2 s_{0}\left[\frac{\left|\Psi_{b}\right| \varepsilon s_{0}^{d} \phi_{e}}{m \pi}\right]^{\frac{1}{2}}$

where $S_{e r}$ is an exfiltration sorptivity coefficient, assumed to be constant for a soil type, and only $K_{s}$ is spatially variable in (A7). This assumption could be justified by the same reason as offered by Bresler and Dagan (1983), $K_{s}$ has the greatest impact on the exfiltration process. As before, $K_{s}$ is also assumed to follow a lognormal distribution. Combining (A2), (A6) and (A7), we then obtain:

$f_{E T}^{*}=\frac{1}{2} S_{e r} K_{s}^{\frac{1}{2}} t^{-\frac{1}{2}}$

Taking the areal average of Eq. (A9), we obtain an equation for the areal average exfiltration capacity:

$\overline{f_{E T}^{*}}=\frac{1}{2} S_{e r} \overline{K_{s}^{\frac{1}{2}}} t^{-\frac{1}{2}}$

The average exfiltration capacity Eq. (A10), depends on time, which makes it inapplicable for continuous modeling, so we need to convert it into time independent form. For this reason, it is assumed that the exfiltration capacity is a function of the volume of cumulative exfiltration only, in analogy with the Time Condensation Approximation (Sherman, 1943) used for infiltration. Then, the cumulative volume of exfiltration can be obtained by integration of (A9) as:

$F_{E T}=S_{e r} K_{S}^{\frac{1}{2}} t^{\frac{1}{2}}$

which means that the areal average of the cumulative volume of exfiltration will be:

$\overline{F_{E T}}=S_{e r} \overline{K_{S}^{\frac{1}{2}}} t^{\frac{1}{2}}$

If we eliminate $t^{\frac{1}{2}}$ between (A12) and (A10) we obtain:

$\overline{f_{E T}^{*}}=\frac{1}{2} \frac{S_{e r}^{2}\left[\overline{K_{s}^{\frac{1}{2}}}\right]^{2}}{\overline{F_{E T}}}$ 
Since $K_{S}$ is assumed to follow a lognormal distribution, following Rogers (1992), $\left[\overline{K_{s}^{\frac{1}{2}}}\right]^{2}$ can be replaced by following relationship

$$
\left[\overline{K_{s}^{\frac{1}{2}}}\right]^{2}=\overline{K_{s}} \exp \left(-\frac{\sigma_{n}^{2}}{4}\right)
$$

where $\sigma_{n}^{2}$ is the variance of logarithm of $K_{s}$. If we now combine (A8) and (A14), and substitute in (A13), we will finally obtain:

$\overline{f_{E T}^{*}}=\alpha \frac{\overline{K_{s}}}{\overline{F_{E T}}} \frac{s_{0}^{2+d} \varepsilon\left|\Psi_{b}\right|}{m}$

where $\alpha=\frac{2 \phi_{e}}{\pi} \exp \left(-\frac{\sigma_{n}^{2}}{4}\right)$ is an estimatable parameter that is related to the variability of saturated hydraulic conductivity, and the dimensionless exfiltration diffusivity $\phi_{e}$. To use (A15) within the REW approach, it is assumed that the spatial average of the cumulative volume of exfiltration is proportional to the pore fraction that is not filled with water, i.e., $\overline{F_{E T}} \approx\left(1-s^{u}\right) y^{u}$, where the initial degree of saturation at the soil surface is replaced by the saturation degree of the entire unsaturated zone. Consequently, we obtain the following exfiltration capacity equation:

$\overline{f_{E T}^{*}}=\alpha \frac{\overline{K_{s}}}{\left(1-s^{u}\right) y^{u}} \frac{\left(s^{u}\right)^{2+d} \varepsilon\left|\Psi_{b}\right|}{m}$

Therefore, the final closure relation, for the combination of bare soil evaporation and transpiration by root water uptake, will be specified as:

$e_{w g}^{u}=\min \left[\left(e_{p}+M \overline{e_{v}}\right) \omega^{u}, \quad \overline{f_{E T}^{*}} \omega^{u}\right]$

where $\overline{e_{v}}$ is spatially averaged transpiration rate at each time step. Since this may not be available in most catchments, it is assumed that $\overline{e_{v}}$ is constant and equal to long-term time average potential rate of transpiration $\overline{e_{p v}}$. By using the ratio $k_{v}=\frac{\overline{e_{p v}}}{\overline{e_{p}}}$, where $\overline{e_{p}}$ is long-term time average rate of potential (soil-surface) evaporation, the probable maximum exfiltration rate, $\left.\overline{f_{E T}}\right|_{\max }$, at each time step, will be expressed by:

$\left.\overline{f_{E T}}\right|_{\max }=e_{p}+M k_{v} \overline{e_{p}}$

and the corresponding closure relation is written as:

$e_{w g}^{u}=\min \left[\left(e_{p}+M k_{v} \overline{e_{p}}\right) \omega^{u}, \quad \overline{f_{E T}^{*}} \omega^{u}\right]$ 


\begin{tabular}{|c|c|c|}
\hline \multicolumn{3}{|c|}{$\begin{array}{l}\text { Nomenclature } \\
\text { Latin symbols }\end{array}$} \\
\hline $\operatorname{vec} A$ & mantle surface with horizontal normal delimiting the REW externally & \\
\hline$A$ & linearisation coefficient for the mass exchange terms & {$\left[T L^{-1}\right]$} \\
\hline$B$ & linearisation coefficient for the mass exchange terms & {$\left[M L^{-3}\right]$} \\
\hline$c$ & pore disconnectedness index & \\
\hline$d$ & diffusivity index & \\
\hline $\bar{d}$ & depth of pore contained in a surface soil layer & {$[L]$} \\
\hline$D$ & diffusivity & {$\left[L^{2} T^{-1}\right]$} \\
\hline $\bar{D}$ & depth of a surface soil layer & {$[L]$} \\
\hline$D_{e}$ & desorption diffusivity & {$\left[L^{2} T^{-1}\right]$} \\
\hline$D I$ & dryness index, or the ratio of annual potential evaporation to annual precipitation & \\
\hline$e$ & water mass exchange per unit surface area divided by water mass density & {$\left[L T^{-1}\right]$} \\
\hline$e_{p}$ & potential evaporation rate from a bare soil surface & {$\left[L T^{-1}\right]$} \\
\hline$\frac{P}{e_{p}}$ & long term time average rate of potential (soil surface) evaporation & {$\left[L T^{-1}\right]$} \\
\hline$\frac{p}{e_{p v}}$ & long-term time average potential rate of transpiration & {$\left[L T^{-1}\right]$} \\
\hline$e_{v}$ & transpiration rate at the point scale & {$\left[L T^{-1}\right]$} \\
\hline$\overline{e_{v}}$ & spatially averaged transpiration rate & {$\left[L T^{-1}\right]$} \\
\hline$f$ & a parameter controlling the exponential decline of transmissivity with depth & {$\left[L^{-1}\right]$} \\
\hline$\overline{f^{*}}$ & spatially averaged infiltration capacity & {$\left[L T^{-1}\right]$} \\
\hline$f_{e}^{*}$ & exfiltration capacity for bare soil evaporation & {$\left[L T^{-1}\right]$} \\
\hline$f_{E T}^{*}$ & evapotranspiration capacity by exfiltration of water from subsurface & {$\left[L T^{-1}\right]$} \\
\hline $\begin{array}{l}\overline{f_{E T}^{*}} \\
f_{M}\end{array}$ & $\begin{array}{l}\text { spatially averaged exfiltration capacity on bare soil evaporation and transpiration by root uptake } \\
\text { macro-porosity factor }\end{array}$ & {$\left[L T^{-1}\right]$} \\
\hline $\bar{F}$ & spatially averaged cumulative volume of infiltration & {$[L]$} \\
\hline$F_{E T}$ & cumulative volume of evapotranspiration by exfiltration & {$[L]$} \\
\hline$\overline{F_{E T}}$ & spatially averaged cumulative volume of evapotranspiration by exfiltration & {$[L]$} \\
\hline$g$ & gravitational acceleration & {$\left[L T^{-2}\right]$} \\
\hline$g_{r}$ & root extraction function & \\
\hline$h_{f}$ & free water content & {$[L]$} \\
\hline$i$ & rainfall intensity & {$\left[L T^{-1}\right]$} \\
\hline$J$ & rate of rainfall input or evaporation & {$\left[L T^{-1}\right]$} \\
\hline$k_{v}$ & ratio of potential rates of transpiration and soil surface evaporation & \\
\hline$K$ & hydraulic conductivity at the point scale & {$\left[L T^{-1}\right]$} \\
\hline $\bar{K}$ & hydraulic conductivity at the catchment scale & {$\left[L T^{-1}\right]$} \\
\hline$K^{B}$ & bulk hydraulic conductivity & {$\left[L T^{-1}\right]$} \\
\hline$K_{s}$ & saturated hydraulic conductivity at the point scale & {$\left[L T^{-1}\right]$} \\
\hline$\overline{K_{s}}$ & mean saturated hydraulic conductivity & {$\left[L T^{-1}\right]$} \\
\hline$l_{i}$ & wetness index at grid point $i$, or $\ln \left(a[\tan \beta]^{-1}\right)_{i}$ & \\
\hline$l^{r}$ & the length of a channel reach & {$[L]$} \\
\hline$L$ & the length of a slope unit & {$[L]$} \\
\hline$M$ & vegetated fraction of land surface, or canopy density & \\
\hline$m$ & pore size distribution index & \\
\hline $\bar{m}$ & constant used in kinematic wave equation (Ichikawa and Shiiba, 2002) & \\
\hline$m^{r}$ & average channel cross sectional area & {$\left[L^{2}\right]$} \\
\hline$N$ & constant used in kinematic wave equation (Ichikawa and Shiiba, 2002) & \\
\hline$n_{m}$ & Manning roughness coefficient & {$\left[T L^{-1 / 3}\right]$} \\
\hline$n$ & shape parameter & \\
\hline$p$ & pressure & {$\left[F L^{-2}\right]$} \\
\hline$P$ & the wetted perimeter & {$[L]$} \\
\hline$q$ & hillslope discharge per unit width & {$\left[L^{2} T^{-1}\right]$} \\
\hline$q_{s}$ & steady flow from saturated zone to channel reach & {$\left[L T^{-1}\right]$} \\
\hline$R$ & first order friction term & {$\left[F T L^{-3}\right]$} \\
\hline
\end{tabular}




\begin{tabular}{|c|c|c|}
\hline & & \\
\hline $\bar{R}$ & the hydraulic radius & {$[L]$} \\
\hline$s$ & the saturation function of unsaturated zone at the point scale & \\
\hline$s^{u}$ & the saturation function of unsaturated zone at the catchment scale & \\
\hline$s_{0}$ & initial degree of saturation in surface boundary layer & \\
\hline$\overline{s_{0}}$ & threshold value of saturation function for macropore flow & \\
\hline$S$ & saturation degree of the entire volume of soil media & \\
\hline $\bar{S}$ & storage volume of a slope unit & {$\left[L^{3}\right]$} \\
\hline$S_{e}$ & exfiltration sorptivity & {$\left[L T^{-0.5}\right]$} \\
\hline$S_{e r}$ & exfiltration sorptivity coefficient & {$\left[L^{0.5}\right]$} \\
\hline$t$ & time & {$[T]$} \\
\hline$t_{r}$ & storm period & {$[T]$} \\
\hline$t_{b}$ & inter-storm period & {$[T]$} \\
\hline$t_{a}$ & climatic period & {$[T]$} \\
\hline$U$ & second order friction term & {$\left[F T^{2} L^{-4}\right]$} \\
\hline $\bar{U}$ & the upslope contributing area of a slope unit & {$\left[L^{2}\right]$} \\
\hline$v$ & velocity of bulk phases & {$\left[L T^{-1}\right]$} \\
\hline $\bar{w}$ & average width of a slope unit & {$[L]$} \\
\hline$w^{r}$ & top width of the channel & {$[L]$} \\
\hline$y$ & average vertical thickness & {$[L]$} \\
\hline$Z$ & average thickness of the subsurface zone & {$[L]$} \\
\hline$z$ & vertical coordinate & {$[L]$} \\
\hline $\bar{z}$ & average water table depth from the ground surface & {$[L]$} \\
\hline$z^{r}$ & average elevation of channel bed from datum & {$[L]$} \\
\hline$z^{s}$ & average elevation of the bottom end of the REW from datum & {$[L]$} \\
\hline Greel & symbols & \\
\hline$\alpha$ & a parameter & \\
\hline $\bar{\alpha}$ & air entry value & {$\left[L^{-1}\right]$} \\
\hline$\beta$ & a parameter & \\
\hline$\delta_{l}$ & the local angle between the reach of the REW $l$ and the reach of the REW under consideration & \\
\hline$\gamma_{c}$ & field capacity & \\
\hline$\gamma_{e}$ & effective porosity & \\
\hline$\gamma^{i}$ & slope angle of the $i$-subregion flow plane with respect to the horizontal plane & \\
\hline$\eta$ & the slope gradient of a slope unit & \\
\hline$\varepsilon$ & soil porosity & \\
\hline $\bar{\lambda}$ & expectation of wetness index $l_{i}$ & \\
\hline$\theta$ & effective volumetric moisture content & \\
\hline$\theta_{0}$ & initial effective volumetric moisture content & \\
\hline$\theta_{1}$ & effective volumetric moisture content at surface of medium & \\
\hline$\theta_{i}$ & initial soil moisture content & \\
\hline$\theta_{r}$ & residual water content & \\
\hline$\theta_{s}$ & saturated soil moisture content & \\
\hline$\rho$ & water mass density & {$\left[M L^{-3}\right]$} \\
\hline$\sigma_{n}^{2}$ & the variance of logarithm of saturated hydraulic conductivity & \\
\hline$\Sigma$ & projection of the total REW surface area onto the horizontal plane & {$\left[L^{2}\right]$} \\
\hline$\xi_{f}$ & Darcy-Weisbach friction factor & \\
\hline$\xi^{r}$ & the length of the main channel reach per unit surface area projection & {$\left[L^{-1}\right]$} \\
\hline$|\psi|$ & soil matric potential head of unsaturated zone at the point scale & {$[L]$} \\
\hline$|\Psi|$ & soil matric potential head of unsaturated zone at the catchment scale & {$[L]$} \\
\hline$\left|\Psi_{b}\right|$ & bubbling pressure head & {$[L]$} \\
\hline$\left|\Psi_{f}\right|$ & soil matric potential head at the wetting front & {$[L]$} \\
\hline$\phi$ & the gravitational potential & \\
\hline$\phi_{e}$ & dimensionless exfiltration diffusivity & \\
\hline$\omega$ & time averaged surface area fraction & \\
\hline
\end{tabular}




\begin{tabular}{ll}
\hline Subscripts and superscripts \\
\hline bot & superscript for the region delimiting the domain of interest at the bottom \\
$l$ & subscript indicating the various REWs within the watershed \\
top & superscript for the atmosphere, delimiting the domain of interest at the top \\
$u, s, c, o, r$ & superscripts indicating subregions within a REW \\
$w, g$ & designate the water and the gaseous phase respectively \\
$j A$ & exchange from the $j$-subregion across the external watershed boundary \\
$e x t$ & exchange from the $j$-subregion across the $l$ th mantle segment \\
$l A$ & \\
\hline
\end{tabular}

Acknowledgements. This research was supported by a DAAD (Deutscher Akademischer Austausch Dienst) Visiting Fellowship which enabled the first author to spend time at University of Potsdam in Germany. The first author also thanks the financial support and hospitality of the University of Illinois at Urbana-Champaign where much of this work was completed. The authors appreciate the valuable comments and constructive criticisms offered by Keith Beven and Hans-Joerg Vogel, which helped to greatly improve the manuscript.

Edited by: K. Roth

\section{References}

Attinger, S.: Generalized coarse graining procedures for flow in porous media, Comp. Geosci., 7, 253-273, 2003.

Beven, K. J.: Towards an alternative blueprint for a physically based digitally simulated hydrologic response modelling system, Hydrol. Processes, 16, 189-206, 2002.

Beven, K. J. and Kirkby, M. J.: A physically-based variable contributing area model of basin hydrology, Hydrol. Sci. Bull., 24(1), 43-69, 1979.

Beven, K. J. and Freer, J. E.: Equifinality, data assimilation, and uncertainty estimation in mechanistic modeling of complex environmental systems using the GLUE methodology, J. Hydrol., 249, 11-29, 2001.

Binley, A., Elgy, J., and Beven, K.: A physically based model for heterogeneous hillslopes. 1. Runoff production, Water Resour. Res., 25(6), 1219-1226, 1989a.

Binley, A., Beven, K., and Elgy, J.: A physically based model for heterogeneous hillslopes. 2. Effective hydraulic conductivities, Water Resour. Res., 25(6), 1219-1226, 1989b.

Blöschl, G. and Zehe, E.: On hydrological predictability, Hydrol. Processes, 19(9), 3923-3929, 2005.

Bras, R. L.: Hydrology: An Introduction to Hydrologic Science, Reading, Mass., USA: Addison-Wesley-Longman, 1990.

Bresler, E. and Dagan, G.: Unsaturated flow in spatially variable fields. 2. Application of water flow models to various fields, Water Resour. Res., 19(2), 421-428, 1983.

Chow, V. T., Maidment, D. R., and Mays, L. W.: Applied Hydrology, McGraw-Hill, Inc, 1988.

Dagan, G.: Flow and Transport in Porous Formations, SpringerVerlag, New York, 1989.

Duffy, C. J.: A two-state integral-balance model for soil moisture and groundwater dynamics in complex terrain, Water Resour. Res., 32(8), 2421-2434, 1996.

Eagleson, P. S.: Climate, soil, and vegetation, 1. Introduction to water balance dynamics, Water Resour. Res., 14(5), 705-712, 1978a.

Eagleson, P. S.: Climate, soil, and vegetation, 3. A simplified model of soil moisture movement in the liquid phase, Water Resour. Res., 14(5), 722-730, 1978b.

Eagleson, P. S.: Climate, soil, and vegetation, 4. The expected value of annual evapotranspiration, Water Resour. Res., 14(5), 731739, $1978 \mathrm{c}$.

Ichikawa, Y. and Shiiba, M.: Lumping of kinematic wave equation considering field capacity, 3rd Int. Conf. on Water Resources and Environmental Research, 1, 61-65, 2002.

Kees, C. E., Band, L. E., and Farthing, M. W.: Effects of Dynamic Forcing on Hillslope Water Balance Models, Technical Report CRSC-TR04-12, Center for Research in Scientific Computation, Department of Mathematics, North Carolina State University, 2004.

Kees, C. E., Farthing, M. W., Band, L. E., and Miller, C. T.: Choices of scale and process complexity in hillslope models, Eos Trans. AGU, 83(47), Fall Meet. Suppl., Abstract H62B-0838, 2002.

Lee, H., Sivapalan, M., and Zehe, E.: Representative Elementary Watershed (REW) approach, a new blueprint for distributed hydrologic modelling at the catchment scale, in: Predictions in ungauged basins: international perspectives on state-of-the-art and pathways forward, edited by: Franks, S. W., Sivapalan, M., Takeuchi, K., and Tachikawa, Y., IAHS Press, Wallingford, Oxon, UK, IAHS Publications 301, Paper 15, 2005 a.

Lee, H., Sivapalan, M., and Zehe, E.: Representative Elementary Watershed (REW) approach, a new blueprint for distributed hydrologic modelling at the catchment scale: the development of closure relations, in: Predicting ungauged streamflow in the mackenzie river basin: today's techniques \& tomorrow's solutions, edited by: Spence, C., Pomeroy, J. W., and Pietroniro, A., Canadian Water Resources Association (CWRA), Ottawa, Canada, 165-218, 2005b.

Lunati, I., Attinger, S., and Kinzelbach, W.: Macrodispersivity for transport in arbitrary nonuniform flow fields: Asymptotic and pre-asymptotic results, Water Resour. Res., 38(10), 1187, doi:10.1029/2001WR001203, 2002.

Maurer, T.: Physikalisch begrundete, zeitkontinuierliche Modellierung des Wassertransports in kleinen landlichen Einzugsgebieten, Universitat Karlsruhe, MitteiLungen Inst. F. Hydrologie u. Wasserwirtschaft, H. 61, Universitat Karlsruhe, 1997.

Mualem, Y.: A new model for predicting the hydraulic conductivity of unsaturated porous media, Water Resour. Res., 12, 513-522, 1976.

Philip, J. R.: General method of exact solution of the concentrationdependent diffusion equation, Aust. J. Phys., 13(1), 1-12, 1960.

Press, W. H., Teukolsky, S. A., Vetterling, W. T., and Flannery, B. P.: Numerical Recipes in FORTRAN, Cambridge University Press, 1992. 
Reggiani, P. and Schellekens, J.: Modelling of hydrological responses: the representative elementary watershed approach as an alternative blueprint for watershed modelling, Hydrol. Processes, 17, 3785-3789, 2003.

Reggiani, P., Sivapalan, M., and Hassanizadeh, S. M.: A unifying framework for watershed thermodynamics: balance equations for mass, momentum, energy and entropy and the second law of thermodynamics, Adv. Water Resour., 22(4), 367-398, 1998.

Reggiani, P., Hassanizadeh, S. M., Sivapalan, M., and Gray, W. G.: A unifying framework for watershed thermodynamics: constitutive relationships, Adv. Water Resour., 23(1), 15-39, 1999.

Reggiani, P., Sivapalan, M., and Hassanizadeh, S. M.: Conservation equations governing hillslope responses: exploring the physical basis of water balance, Water Resour. Res., 36(7), 1845-1863, 2000.

Reggiani, P., Sivapalan, M., Hassanizadeh, S. M., and Gray, W. G.: Coupled equations for mass and momentum balance in a stream network: theoretical derivation and computational experiments, Proc. R. Soc. Lond., 457, 157-189, 2001.

Robinson, J. S. and Sivapalan, M.: Catchment-scale runoff generation model by aggregation and similarity analyses, Hydrol. Processes, 9, 555-574, 1995.

Robock, A., Vinnikov, K. Y., Srinivasan, G., Entin, J. K., Hollinger, S. E., Speranskaya, N. A., Liu, S., and Namkhai, A.: The Global Soil Moisture Data Bank. Bull. Amer. Meteor. Soc., 81, 12811299, 2000.

Rogers, A. D.: The development of a simple infiltration capacity equation for spatially variable soils, B.E (Honours) thesis, Department of Civil and Environmental Engineering, The University of Western Australia, 1992.

Schäfer, D.: Bodenhydraulische Eigenschaften eines Kleineinzugsgebiets - Vergleich und Bewertung unterschiedlicher Verfahren, $\mathrm{PhD}$ dissertation, Institute of Hydromechanics, University of Karlsruhe, Germany, 1999.

Schulz, K., Seppelt, R., Zehe, E., Vogel, H. J., and Attinger, S.: Importance of spatial structures in advancing hydrological sciences, Water Resour. Res., 42(3), W03S03, doi:10.1029/2005WR004301, 2006.

Sherman, L. K.: Comparison of F-curves derived by the methods Sharp and Holtan and of Sherman and Mayer, Trans. Am. Geophys. Union, 24, 465-467, 1943.

Sivapalan, M. and Wood, E. F.: Spatial heterogeneity and scale in the infiltration response of catchments, in: Scale problems in hydrology, edited by: Gupta, V. K., Rodríguez-Iturbe I., and Wood, E. F., Reidel Publ., Dordrecht, pp. 81-106, 1986.

Sivapalan, M.: Linking hydrologic parameterizations across a range of spatial scales: hillslope to catchment to region, IAHS Publ. 212, Proc. Yokohama Symposium, 115-123, 1993.
Sivapalan, M., Takeuchi, K., Franks, S.W., Gupta, V.K., Karambiri, H., Lakshmi, V., Liang, X., McDonnell, J.J., Mendiondo, E.M., O'Connel, P.E., Oki, T., Pomeroy, J.W., Schertzer, D., Uhlenbrook, S., and Zehe, E.: IAHS decade on Predictions of Ungauged Basins (PUB): Shaping an exciting future for the hydrological sciences, Hydrol. Sci. J., 48(6), 857-879, 2003.

Sloan, P. G. and Moore, I. D.: Modeling subsurface stormflow on steeply sloping forested watersheds, Water Resour. Res., 20(12), 1815-1822, 1984.

van Genuchten, M. T.: A closed-form equation for predicting the hydraulic conductivity of unsaturated soils, Soil Sci. Soc. Am. Jour., 44, 892-898, 1980.

Viney, N. R. and Sivapalan, M.: A framework for scaling of hydrologic conceptualisations based on a disaggregation-aggregation approach, Hydrol. Processes, 18, 1395-1408, 2004.

Vogel, H. J. and Roth, K.: Moving through scales of flow and transport in soil, J. Hydrol., 272, 95-106, 2003.

Wagener, T., McIntyre, N., Lees, M. J., Wheater, H. S., and Gupta, H. V.: Towards reduced uncertainty in conceptual rainfall-runoff modelling: Dynamic identifiability analysis, Hydrol. Processes, 17, 455-476, 2003.

Western, A. W. and Grayson, R. B.: The Tarrawarra data set: soil moisture patterns, soil characteristics and hydrological flux measurements, Water Resour. Res. 34(10), 2765-2768, 1998.

Zehe, E., Maurer, T., Ihringer, J., and Plate, E.: Modeling water flow and mass transport in a Loess catchment, Phys. Chem. Earth (B), 26, 7-8, 487-507, 2001.

Zehe, E. and Blöschl, G.: Predictability of hydrologic response at the plot and catchment scales - the role of initial conditions, Water Resour. Res., 40(10), W10202, doi:10.1029/2003WR002869, 2004.

Zehe, E., Lee, H., and Sivapalan, M.: Derivation of closure relations and commensurate state variables for mesoscale hydrological models using dynamical upscaling, in: Predictions in ungauged basins: international perspectives on state-of-the-art and pathways forward, edited by: Franks, S. W., Sivapalan, M., Takeuchi, K., and Tachikawa, Y., IAHS Press, Wallingford, Oxon, UK, IAHS Publications 301, Paper 14, 2005 a.

Zehe, E., Becker, R., Bardossy, A., and Plate, E.: Uncertainty of simulated catchment runoff response in the presence of threshold processes: Role of initial soil moisture and precipitation, J. Hydrol., 315, 183-202, 2005b.

Zehe, E., Lee, H., and Sivapalan, M.: Dynamical process upscaling for deriving catchment scale state variables and constitutive relations for meso-scale process models, Hydrol. Earth Syst. Sci., 10, 981-996, 2006, http://www.hydrol-earth-syst-sci.net/10/981/2006/. 